\title{
Cooling of neutron stars. Hadronic model
}

\author{
D. Blaschke ${ }^{1,2,3}$, H. Grigorian ${ }^{1,4}$, and D. N. Voskresensky ${ }^{5,6}$ \\ ${ }^{1}$ Fachbereich Physik, Universität Rostock, Universitätsplatz 1, 18051 Rostock, Germany \\ e-mail: david.blaschke@physik.uni-rostock.de \\ ${ }^{2}$ Bogoliubov Laboratory for Theoretical Physics, Joint Institute for Nuclear Research, 141980 Dubna, Russia \\ 3 Fakultät für Physik, Universität Bielefeld, Universitätsstrasse, 33615 Bielefeld, Germany \\ 4 Department of Physics, Yerevan State University, Alex Manoogian Str. 1, 375025 Yerevan, Armenia \\ e-mail: hovik@darss.mpg.uni-rostock.de \\ 5 Gesellschaft für Schwerionenforschung mbH, Planckstr. 1, 64291 Darmstadt, Germany \\ ${ }^{6}$ Moscow Institute for Physics and Engineering, 115409 Moscow, Russia \\ e-mail: d.voskresensky@gsi.de
}

Received 8 Mars 2004 / Accepted 17 June 2004

\begin{abstract}
We study the cooling of isolated neutron stars. The main cooling regulators are introduced: equation of state (EoS), thermal transport, heat capacity, neutrino and photon emissivity, superfluid nucleon gaps. The neutrino emissivity includes the main processes. A strong impact of medium effects on the cooling rates is demonstrated. Taking into account medium effects on reaction rates and on nucleon superfluid gaps modern experimental data can be well explained.
\end{abstract}

Key words. dense matter - equation of state - neutrinos - stars: evolution - stars: neutron - stars: pulsars: general

\section{Introduction}

The Einstein Observatory was the first that started the experimental study of surface temperatures of isolated neutron stars (NS). Upper limits for some sources have been found. Then ROSAT offered first detections of surface temperatures. At present X-ray data come from Chandra and XMM/Newton. Appropriate references to the modern data can be found in recent works by Tsuruta et al. (2002), Tsuruta (2004), Kaminker et al. (2002), and Yakovlev et al. (2004a), devoted to the analysis of the new data. More upper limits and detections are expected from satellites planned to be launched in the nearest future. In general, the data can be separated into three groups. Some data show very slow cooling of objects, others demonstrate intermediate cooling and some show very rapid cooling. Now we are in a position to carefully compare the data with existing cooling calculations.

\section{Existing NS cooling scenarios}

Let us briefly point out some important achievements on the way to the present understanding of the NS cooling problem. The theoretical study of NS cooling was started long ago with the pioneering works of Tsuruta \& Cameron (1965) and Bahcall \& Wolf (1965). It has been argued that the one-nucleon process $\mathrm{n} \rightarrow \mathrm{p} e \bar{v}$, the so called direct Urca (DU) process, is forbidden up to sufficiently high density and the main rôle is played by the two-nucleon processes such as nn $\rightarrow$ npe $\bar{v}$ and $\mathrm{np} \rightarrow \mathrm{pp} e \bar{v}$, the so called modified Urca (MU) processes.
As the result of many studies the so called Standard scenario of cooling emerged. It includes the neutrino cooling stage for $t \lesssim 10^{5} \mathrm{yr}$ and the photon cooling era for $t \gtrsim 10^{5} \mathrm{yr}$. The MU processes and the nucleon-nucleon bremsstrahlung (NB) processes, as $\mathrm{nn} \rightarrow \mathrm{nn} v \bar{v}$ and $\mathrm{np} \rightarrow \mathrm{np} v \bar{v}$, were carefully recalculated by Friman \& Maxwell (1979) using the free one-pion exchange. The emissivity of the most efficient channel of the MU process $n n \rightarrow$ npe $\bar{v}$ is given by

$\varepsilon_{v}[\mathrm{MU}] \sim 10^{22}\left(m_{\mathrm{MU}}^{*}(n)\right)^{4}\left[\frac{n_{\mathrm{e}}}{n_{0}}\right]^{1 / 3} \xi_{\mathrm{nn}} \xi_{\mathrm{pp}} T_{9}^{8}, \frac{\mathrm{erg}}{\mathrm{cm}^{3} \mathrm{~s}}$,

where $T_{9}=T / 10^{9} \mathrm{~K}$ is the temperature, $n_{\mathrm{e}}$ the electron density and $n_{0} \simeq 0.16 \mathrm{fm}^{-3}$ the nuclear saturation density. $\left(m_{\mathrm{MU}}^{*}(n)\right)^{4}=$ $\left(m_{\mathrm{n}}^{*} / m_{\mathrm{N}}\right)^{3}\left(m_{\mathrm{p}}^{*} / m_{\mathrm{N}}\right)$, where $m_{\mathrm{n}}^{*}$ and $m_{\mathrm{p}}^{*}$ are the effective neutron and proton masses, $m_{\mathrm{N}}$ is the nucleon mass in vacuum, and $n$ is the nucleon density; f.i. $\left(m_{\mathrm{MU}}^{*}(n)\right)^{4}\left(n_{\mathrm{e}} / n_{0}\right)^{1 / 3} \sim 0.1$ for $n \sim n_{0}$.

After Migdal (1959) it became clear that NS in the late cooling stage for $T<T_{\text {cn }}$ and $T<T_{\text {cp }}$ are neutron and proton superfluids. The nucleon superfluidity was incorporated in the Standard scenario by Maxwell (1979), who used relevant combinations of the suppression factors

$\xi_{i i} \simeq \exp \left(-\Delta_{i i} / T\right), \quad T<T_{\mathrm{c} i}$

namely $\xi_{\mathrm{nn}} \xi_{\mathrm{pp}}$ for the emissivity of MU (nn $\rightarrow$ npe $\left.\bar{v}\right)$ and NB $(\mathrm{np} \rightarrow \mathrm{np} v \bar{v})$ processes, $\xi_{\mathrm{nn}}^{2}$ for NB (nn $\left.\rightarrow \mathrm{nn} v \bar{v}\right), \xi_{\mathrm{pp}}^{2}$ for $\mathrm{MU}$ (np $\rightarrow$ ppe $\bar{v}$ ) processes for $T<T_{\mathrm{cn}}$ and $T<T_{\mathrm{cp}}$, where $\Delta_{i i}$ is the gap and $i=\mathrm{n}$ or $\mathrm{p}$. 
Typically the NB emissivity is smaller by an order of magnitude than that for MU. The prefactor $\sim 10^{20}-10^{21}$ and the temperature dependence $T_{9}^{8}$ are typical for the phase space volume of two-nucleon processes (without inclusion of medium effects, see below).

The first cooling calculations were based on the assumption of an isothermal core. The relevance of the thermal conductivity for the first $10^{2}-10^{3} \mathrm{yr}$ was demonstrated by Nomoto \& Tsuruta (1981). A discussion of the achievements of the early works can be found in the review by Tsuruta (1979) and in the book by Shapiro \& Teukolsky (1983).

The Standard scenario makes it possible to explain the slow cooling but fails to describe the intermediate cooling and the fast cooling of some neutron stars. To explain the latter various efficient direct Urca-like processes have been suggested: the proper DU process, reinvestigated by Lattimer et al. (1991) and the DU process on hyperons (HDU), see the same work; pion condensation (PU) processes by Maxwell et al. (1977); kaon condensation (KU) processes by Brown et al. (1988), Tatsumi (1988), similar to PU; and quark DU processes (QDU) by Iwamoto (1982). All these processes show up for baryon densities larger than the corresponding critical ones $n>n_{\mathrm{c}}^{\mathrm{DU}}, n_{\mathrm{c}}^{\mathrm{PU}}, n_{\mathrm{c}}^{\mathrm{KU}}, n_{\mathrm{c}}^{\mathrm{HDU}}, n_{\mathrm{c}}^{\mathrm{QDU}} \sim 1.5-6 n_{0}$ according to different model-dependent estimates. Roughly

$\varepsilon_{v}[\mathrm{DU}] \sim 10^{27}\left(m_{\mathrm{DU}}^{*}\right)^{2}\left[\frac{n_{e}}{n_{0}}\right]^{1 / 3} T_{9}^{6} \min \left[\xi_{\mathrm{nn}}, \xi_{\mathrm{pp}}\right] \frac{\mathrm{erg}}{\mathrm{cm}^{3} \mathrm{~s}}$,

where $\left(m_{\mathrm{DU}}^{*}\right)^{2}=\left(m_{\mathrm{n}}^{*} m_{\mathrm{p}}^{*}\right) / m_{\mathrm{N}}^{2}$. The factors $10^{26}-10^{29}$ and the behavior $\sim T_{9}^{6}$ are typical for all the above mentioned oneparticle processes. Thus the Standard scenario+exotics has been considered as a scenario with minimum exotics: some stars have $n<n_{\mathrm{c}}$ and cool slowly whereas some have $n>n_{\mathrm{c}}$ in a part of their interiors and cool very fast.

However, one type of non-exotic processes was completely forgotten already in the Standard scenario without any justification. These are the so called neutron pair breaking and formation $(n \mathrm{PBF})$ and proton pair breaking and formation $(p \mathrm{PBF})$ processes, as they were named by Schaab et al. (1997). The emissivity of the $n$ PBF process was first calculated by Flowers et al. (1976) for the case of the $1 \mathrm{~S}_{0}$ neutron pairing. However, their asymptotic expression for the emissivity $\varepsilon[n \mathrm{PBF}] \sim$ $10^{20} T_{9}^{7} \exp \left(-2 \Delta_{\mathrm{nn}} / T\right)$ for $T \ll \Delta_{\mathrm{nn}}$, as follows from expression $(1 \mathrm{~b})$ of their work and from their rough asymptotic estimate of the integral (see below (13b)), shows neither the large one nucleon phase space factor $\left(\sim 10^{29}\right)$ nor the appropriate temperature behaviour (note that the full analytic expression of this work is quite correct). The numerical underestimation of the rate by an order of magnitude and the very rough asymptotic expression used by Flowers et al. (1976) became the reason that this important result was overlooked for many years. Voskresensky \& Senatorov (1987) rediscovered the $n$ PBF process $\mathrm{n} \rightarrow \mathrm{n} v \bar{v}$ and introduced the $p$ PBF process $\mathrm{p} \rightarrow \mathrm{p} v \bar{v}$ with correct asymptotic behavior of the emissivity

$$
\begin{aligned}
\varepsilon_{v}[\mathrm{PBBF}] \sim & 10^{29} m_{\mathrm{iPBF}}^{*}\left[\frac{p_{F i}(n)}{p_{F n}\left(n_{0}\right)}\right]\left[\frac{\Delta_{i i}}{\mathrm{MeV}}\right]^{7}\left[\frac{T}{\Delta_{i i}}\right]^{1 / 2} \\
& \times \xi_{i i}^{2} \frac{\mathrm{erg}}{\mathrm{cm}^{3} \mathrm{~s}}
\end{aligned}
$$

for $T \ll \Delta_{i i}, p_{F i}(n)$ is the neutron/proton Fermi momentum, $i=\mathrm{n}, \mathrm{p}, m_{\mathrm{iPBF}}^{*}=m_{i}^{*} / m_{\mathrm{N}}$. Equation (4) shows a huge onenucleon phase space factor and a quite moderate $T$ dependence of the pre-factor (note that the value of the pre-factor in case of $1 \mathrm{~S}_{0}$ neutron pairing evaluated by Voskresensky \& Senatorov 1987 contains a mistake that however yields only a non-essential correction factor of order one to their final result); a larger uncertainty comes from the not too well known medium-modified vertices (see the discussion by Voskresensky 2001 and the corrected expressions there). Medium modifications of vertices (cf. Ward-Takahashi identity) have been incorporated in all processes. This is a very important point since medium modified vertices yield an enhancement factor up to $10^{2}$ for $p$ PBF compared with the result one would get using vacuum vertices. This factor $\sim 10^{2}$ arises since the process may occur through $\mathrm{nn}^{-1}$ and $\mathrm{ee}^{-1}$ correlations, -1 symbolizes the particle hole, with subsequent production of $v \bar{v}$ from the $\mathrm{n}$ and e rather than from a strongly suppressed vacuum vertex of p $v \bar{v}$ (see Voskresensky \& Senatorov 1987; Voskresensky et al. 1998; Leinson 2000; Voskresensky 2001). The efficiency of the PBF rates was related by Voskresensky \& Senatorov (1987) to the value of the pairing gap and it was indicated that these processes can play an important rôle in the cooling scenario. Note that if one takes the medium effects into account the estimate (4) is roughly valid for all $1 \mathrm{~S}_{0}$ neutron and proton pairings as well as for the $3 \mathrm{P}_{2}$ neutron pairing, except the specific case when the projection of the pair momentum onto the quantization axis is $\left|m_{\mathrm{J}}\right|=2$. In the latter case, where the gap has zeros in some points of the Fermi surface, the emissivity would not be exponentially suppressed (cf. Voskresensky \& Senatorov 1987). The conditions, however, under which such a possibility might be realized, are not clear.

The inclusion of the PBF processes in the cooling code was first accomplished by Schaab et al. (1997) (where also for the first time the reference to the pioneering work of Flowers et al. 1976 appeared) with the result that a variation of the gaps makes it possible to develop a scenario for intermediate cooling covering the region of both slow and intermediate cooling. Some subsequent studies rediscovered the $p$ PBF process with vacuum vertices thus ignoring medium effects but including relativistic corrections to the vacuum vertex. The latter corrections yield an enhancement of order 10 rather than the mentioned $10^{2}$ and are therefore of minor importance compared to the larger contribution of medium effects. The $p$ PBF emissivity evaluated with vacuum vertex (with relativistic corrections) was implemented into their cooling code by Yakovlev et al. (2004a). Their result for this process should be still enhanced by at least a factor $\gtrsim 10$ to account for medium effects.

Medium effects essentially modify not only the $p \mathrm{PBF}$ emissivity but they correct also the contributions from all other processes. They were first included into the emissivities of different processes by Voskresensky \& Senatorov (1984, 1986, 1987), (see also Migdal et al. 1990 and a more recent review, Voskresensky 2001). It was shown that the main contribution to the MU process actually comes from the pion channel of the reaction $\mathrm{nn} \rightarrow \mathrm{np} e \bar{v}$, where $e \bar{v}$ are radiated from the intermediate pion and the $\mathrm{NN}^{-1}$ loop rather than from the nucleon of the leg of the reaction. Moreover, due to the so called 
pion softening (medium modification of the pion propagator) the matrix elements of the MU process are further enhanced with increasing density towards the critical point of pion condensation. Thus the corrected MU process has been called the medium modified Urca (MMU) and the corrected NB process has been called MNB. The full expressions for the emissivities of the MMU and MNB processes were derived with the help of the Landau-Migdal Fermi liquid approach. These are complicated functions of several Landau-Migdal parameters and the effective pion gap and look rather cumbersome; details can be found in the reviews by Migdal et al. (1990), and Voskresensky (2001). Roughly, the emissivity (1) acquires a factor (mainly due to the pion decay channel of MMU)

$\frac{\varepsilon_{v}[\mathrm{MMU}]}{\varepsilon_{v}[\mathrm{MU}]} \sim 10^{3}\left(n / n_{0}\right)^{10 / 3} \frac{\Gamma^{6}(n)}{\omega^{* 8}(n)}$,

where the prefactor $\left(n / n_{0}\right)^{10 / 3}$ arises from the phase space volume, $\Gamma(n)=1 /\left[1+C\left(n / n_{0}\right)^{1 / 3}\right], C \simeq 1.4-1.6$, is the proper nucleon-nucleon correlation factor, which appears in the vertices due to the strong interaction, being expressed through the Landau-Migdal parameters (in other words $\Gamma(n)$ enforces the Ward-Takahashi identity). Note that the function $\Gamma$ introduced above is an averaged quantity. Actually the correlation factors depend on the energy-momentum transfer, being different for vertices connected to the weak coupling $\left(\Gamma_{w-\mathrm{s}}\right.$ is rather close to unity) and for vertices related to the pure strong coupling $\left(\Gamma_{\mathrm{s}}\right.$ is slightly less than the above introduced factor $\Gamma$, $\left.\Gamma^{6}=\Gamma_{\mathrm{w}-\mathrm{s}}^{2} \Gamma_{\mathrm{s}}^{4}\right) . \omega^{*}(n)$ is the so called effective pion gap. The latter has the meaning of an effective pion mass at finite momentum transfer in the given reaction where $k=p_{F n}$, namely $\omega^{* 2}(n)=-D^{-1}\left(\omega=0, k=p_{F n}, n\right)=m_{\pi}^{2}+p_{F n}^{2}+\Pi(\omega=0, k=$ $\left.p_{F n}, n\right)$, where $D(\omega, k)$ is the pion quasiparticle Green function and $\Pi(\omega, k)$ is the pion self-energy, extracted from the microscopic analysis and from the comparison with the experimental data on atomic nuclei, (see Migdal et al. 1990).

The quantity $\omega^{* 2}(n)$ replaces the value $\left(m_{\pi}^{2}+p_{F n}^{2}\right)$ in the case of the free pion propagator used for the calculation of the MU process by Friman \& Maxwell (1979). Thus the squared amplitude of the nucleon-nucleon interaction contains $\sim \Gamma^{2} / \omega^{* 2}$ instead of $\sim 1 /\left[m_{\pi}^{2}+p_{F n}^{2}\right]$ for the free one pion exchange used by Friman \& Maxwell (1979). Here we suppressed a smaller contribution of a local Landau-Migdal interaction, being corrected by $N N^{-1}$ loops, (cf. Voskresensky \& Senatorov 1986, 1987; Migdal et al. 1990).

The density dependencies of the correlation factor and the effective pion gap are presented in Fig. 1, (for details see Migdal et al. 1990; Schaab et al. 1997; Voskresensky 2001). We see that vertices are rather strongly suppressed (and this suppression increases with the density) but the softening of the pion mode is enhanced $\left(\omega^{* 2}<m_{\pi}^{2}\right)$ for $n \gtrsim 0.5-0.8 n_{0}$. Such a behavior is motivated both theoretically and by the analysis of nuclear experiments, (see Migdal et al. 1990; Ericson $\&$ Weise 1988). Even with full microscopic calculations the functions $\Gamma(n)$ and $\omega^{*}(n)$ contain large uncertainties. These uncertainties come mainly from simplifications inherent in the Landau-Migdal approach to nuclear forces where the LandauMigdal parameters are constants. In reality they may slowly depend on the energy-momentum, neutron to proton ratio and



Fig. 1. The nucleon-nucleon correlation factor $\Gamma$ and the squared effective pion gap $\omega^{* 2}$ as a function of the baryon density with pion condensation (branches 1a, 2, 3) and without (1a, 1b).

the density. To take these uncertainties into account we allow for a variation of the parameters within wide limits. In Fig. 1, curve 1a shows the behavior of the pion gap for $n<n_{\mathrm{c}}^{\mathrm{PU}}$. The value $n_{\mathrm{c}}^{\mathrm{PU}}$ depends on different rather uncertain parameters (we further assume $n_{\mathrm{c}}^{\mathrm{PU}} \simeq 3 n_{0}$ ), (cf. discussion by Migdal et al. 1990). Curve $1 \mathrm{~b}$ demonstrates the possibility of saturation of pion softening and the absence of pion condensation for $n>n_{\mathrm{c}}^{\mathrm{PU}}$ (this possibility could be realized, e.g., if the Landau-Migdal parameters increased with the density). Curves 2 and 3 demonstrate the possibility of pion condensation for $n>n_{\mathrm{c}}^{\mathrm{PU}}$. The continuation of branch 1a for $n>n_{\mathrm{c}}^{\mathrm{PU}}$ (branch 2) demonstrates the reconstruction of the pion dispersion relation on the ground of the condensate state. Here for simplicity we do not distinguish between $\pi^{0}, \pi^{ \pm}$condensations, (see Voskresensky \& Senatorov 1984; Migdal et al. 1990) and the so called alternative-layer-structure, including both types of condensates, (see Umeda et al. 1994). In agreement with a general trend known in condensed matter physics, fluctuations dominate in the vicinity of the critical point of the phase transition and die out far below and above the critical point (see curves 1a, 2). The jump from branch 1a to branch 3 is due to the first order phase transition to the $\pi$ condensation, (see discussion of this point by Voskresensky \& Mishustin 1982; Migdal et al. 1990). Branch 3 yields the amplitude of the pion condensate mean field for $n>n_{\mathrm{c}}^{\mathrm{PU}}$. The observation that the pion condensation appears as a first order phase transition needs a comment. With first order phase transitions in systems with several charged species one associates the possibility of a mixed phase, (Glendenning 1992). The emissivity is increased within the mixed phase since efficient DU-like processes due to nucleon re-scattering on the new-phase droplets are possible. However, Voskresensky et al. (2002), and Maruyama et al. (2003) demonstrated that the mixed phase, if it exists, is probably realized only in a narrow density interval due to charge screening effects. Therefore, we further disregard the possibility of the mixed phase to simplify the consideration. We also disregard the change in the equation of state (EoS), assuming that the phase transition is rather weak. 
The studies of Voskresensky \& Senatorov $(1984,1986)$ suggest that the difference in the surface temperatures of various compact objects could be explained by the assumption that the objects have different masses and thus different density profiles and different cooling rates, according to the argumentation above.

The most precise measurement of NS masses comes from the binary pulsar system PSRB1913+16, where for the more massive NS a value of $M=1.4411 \pm 0.0007 M_{\odot}$ was obtained (Taylor \& Weisberg 1989). The approximate coincidence of this value with the present statistical average value for masses of binary pulsars $M \approx 1.35 \pm 0.04 M_{\odot}$ (Thorsett \& Chakrabarty 1999) motivated some authors to conjecture that all NS masses should be very close to $1.4 M_{\odot}$. A recent measurement (Lyne et al. 2004) of the NS mass $M \simeq 1.25 M_{\odot}$ in JC 737-3C 3J does not confirm the above conjecture. NS masses can vary within some limits. Thus the argumentation, (cf. Voskresensky \& Senatorov 1986) that slow to fast cooling is explained by different masses of the corresponding objects seems rather natural. Many other in-medium reaction channels have been studied. The particular rôle of medium effects in NS cooling has been reviewed by Migdal et al. (1990) and more recently by Voskresensky (2001). The pion softening with increasing baryon density and the subsequent pion condensation are nowadays reproduced not only by microscopic models of the polarization of the baryonic medium (Migdal et al. 1990; Suzuki et al. 1999), but also by detailed variational calculations of Akmal et al. (1998), yielding $n_{\mathrm{c}}^{\mathrm{PU}} \sim 1.5-3 n_{0}$ for the $\pi^{0}$ and charged $\pi^{-}$condensates. We repeat here the conclusion of the series of above mentioned studies that only due to the enhancement of medium polarization with the baryon density may the pion condensate set in and it seems thereby not justified to ignore the softening effects for $n<n_{\mathrm{c}}^{\mathrm{PU}}$, and suddenly switch on the condensate for $n>n_{\mathrm{c}}^{\mathrm{PU}}$. To avoid a possible misunderstanding we should notice that uncertainties in the knowledge of the NN interaction at high density may still allow the alternative possibility that pion condensation is absent and that even repulsion occurs instead of pion softening. We study all these possibilities by comparing the corresponding cooling curves with the data.

In-medium modifications of different processes including MU, NB, PFB, PU and DU reactions were implemented in the cooling code by Schaab et al. (1997) with the conclusion that the data existing up to that time could be explained assuming different masses of the sources. The statement was demonstrated on different models for the equation of state (EoS) with and without superfluidity effects. However, the best fit to the whole set of data was not worked out. E.g., the slow cooling object PSR 1055-52 was not fitted. As we show below this cannot be done without a fit of the $1 \mathrm{~S}_{0} \mathrm{n}$ and $\mathrm{p}$ and the $3 \mathrm{P}_{2} \mathrm{n}$ gaps, their absolute values and density dependencies. The additional assumption of internal heating has been considered as a possibility to fit hot sources appropriately. Vortex creep heating is physically necessary, e.g., to explain glitch data. The possibility to fit the data on hot sources by including the heating effect was demonstrated by Tsuruta (2004, and references therein). Although in reality probably both medium effects and internal heating are in operation, in order not to complicate matters by introducing extra assumptions, we further disregard the heating effect, thus focusing on the role of medium effects. One may estimate the possible importance of the heating effect from Fig. 1 of the paper by Tsuruta 2004. The inclusion of different medium effects (see Voskresensky 2001) makes it possible to develop a general Nuclear medium cooling scenario describing all the cooling data and covering the gap between the Standard and the Standard+exotics scenarios. The works of Blaschke et al. $(2000,2001)$ considered the possibility of color superconducting pure quark and hybrid stars, and discussed the peculiarities of these objects.

The most recent data have been included in the analysis by Kaminker et al. (2002), Yakovlev et al. (2004a), Tsuruta et al. (2002), and Tsuruta (2004). Medium effects in the emissivity were disregarded. The cooling scenario based on the inclusion of the DU process (Standard+exotics $D U$ ) was worked out by Kaminker et al. (2002) and Yakovlev et al. (2004a). Their result is that the neutron $3 \mathrm{P}_{2}$ gap should be strongly suppressed since otherwise one could not explain the slow cooling objects. This assumption is supported by modern microscopic calculations of the $3 \mathrm{P}_{2}$ gap including medium effects (Schaab et al. 1996; Lombardo \& Schulze 2001; Schwenk \& Friman 2004). On the other hand, according to Kaminker et al. (2002) and Yakovlev et al. (2004a) the $1 \mathrm{~S}_{0}$ proton gap should be enhanced to smoothen the transition from the slow cooling to the rapid cooling. Only switching on the DU process for $M>1.358 M_{\odot}$ in their EoS model makes it possible to explain the intermediate cooling and rapid cooling of some objects. The transition from slow cooling to rapid cooling occurs in a narrow window of masses.

This Standard+exotics DU scenario of Yakovlev et al. can be criticized as follows, (see also Tsuruta et al. 2002; Tsuruta 2004). i) The most elaborated model of the variational theory EoS of Akmal et al. (1998) permits the DU process only for rather high densities $n>n_{\mathrm{c}}^{\mathrm{DU}} \simeq 5.19 n_{0}$ and $M \geq$ $1.839 M_{\odot}$, whereas Yakovlev et al. (2004a) used a model of the EoS where the DU process starts for $n>n_{\mathrm{c}}^{\mathrm{DU}} \simeq 2.8 n_{0}$ $\left(M>1.358 M_{\odot}\right)$. It seems doubtful that the DU reaction might "know" that it should start exactly, when the NS mass is approximately $1.4 M_{\odot}$, which is very close to the average value of the NS mass measured in NS binaries. The NS mass is governed by the full NN interaction whereas the value of the proton fraction, being responsible for the critical density of the DU reaction, is governed by the part of the interaction related to the symmetry energy. In the light of the recent observation of an object with $M \simeq 1.25 M_{\odot}$ the above assumption seems even more unlikely. If the DU process occurred at $M>1.839 M_{\odot}$ as follows from the EoS by Akmal et al. (1998), the model of Yakovlev et al. (2004a) based on the opening of the DU channel would fail. Indeed, it would mean that the majority of isolated NS has masses $M>1.839 M_{\odot}$. The latter sounds quite unrealistic. ii) Yakovlev et al. (2004a) introduced by hand a large proton gap. However, there are indications from Schulze et al. (1996) that medium effects should suppress the proton gap. Moreover, Takatsuka \& Tamagaki (1997) demonstrated that proton and neutron superfluidities must be very weak at the densities when the DU process becomes operative. iii) As we argued, the $p \mathrm{PBF}$ emissivity should be enhanced by 
an order of magnitude by medium effects, which is not taken into account. iv) The MU and the NB processes are significantly affected by medium effects (should be replaced by MMU and MNB), which is not incorporated.

In studies of Tsuruta et al. (2002) and Tsuruta (2004) the Standard+exotics $P U$ scenario (based on the inclusion of the PU process for $n>n_{\mathrm{c}}^{\mathrm{PU}} \simeq 2.5 n_{0}$ and $4 n_{0}$, respectively) was used. In general their slow cooling curves are below those of Yakovlev et al. (2004a). The hot object PSR 1055-52 cannot be described properly if internal heating is not included (see Fig. 1 b of Tsuruta 2004, dot-dashed curve), similar to the result of Schaab et al. (1997). The transition from slow cooling to rapid cooling is here due to the PU process. Again the model assumes that the value of the transition density for the nonstandard process (here pion condensation) is very close to the value of the central density of a "canonical" star of $M \simeq 1.4 M_{\odot}$ in order to have all NS masses very close to the value of $1.4 M_{\odot}$. The latter seems rather unlikely in the light of the recent measurement of a NS star mass $M \simeq 1.25 M_{\odot}$. The medium effects are disregarded for simplicity and thus the above points iii), iv) remain uncorrected.

The need to include in-medium effects into the NS cooling problem is rather obvious. It is based on the whole experience of condensed matter physics and of nuclear physics, and it is called for by the heavy ion collision experiments, (Migdal et al. 1990; Voskresenksy 2001; Ivanov et al. 2001; Wambach 2003). Their relevance for the NS cooling scenario was demonstrated by Schaab et al. (1997). Below we present cooling calculations of NS based on the Nuclear medium cooling scenario, (Voskresensky 2001). We remove the above shortcomings and show the relevance of medium effects on the reaction rates and superfluid gaps by demonstrating the possibility of fitting the whole set of cooling data available today. Uncertainties in quantities characterizing medium effects at high density allow a broad variation of parameters of the model which we realize by using different curves for $\omega^{*}(n)$.

\section{EoS and structure of NS interior, crust, and surface}

\subsection{NS interior}

We will exploit the EoS of Akmal et al. (1998) (specifically the Argonne $V 18+\delta v+U I X^{*}$ model), which is based on the most recent models for the nucleon-nucleon interaction with the inclusion of a parameterized three-body force and relativistic boost corrections. Actually we adopt a simple analytic parameterization of this model given by Heiselberg \& Hjorth-Jensen (1999) (HHJ). The latter uses the compressional part with the compressibility $K \simeq 240 \mathrm{MeV}$, and a symmetry energy fitted to the data around nuclear saturation density, and smoothly incorporates causality at high densities. The density dependence of the symmetry energy is very important since it determines the value of the threshold density for the DU process. The HHJ EoS fits the symmetry energy to the original Argonne $V 18+\delta v+U I X^{*}$ model yielding $n_{\mathrm{c}}^{\mathrm{DU}} \simeq 5.19 n_{0}$ $\left(M_{\mathrm{c}}^{\mathrm{DU}} \simeq 1.839 M_{\odot}\right)$.

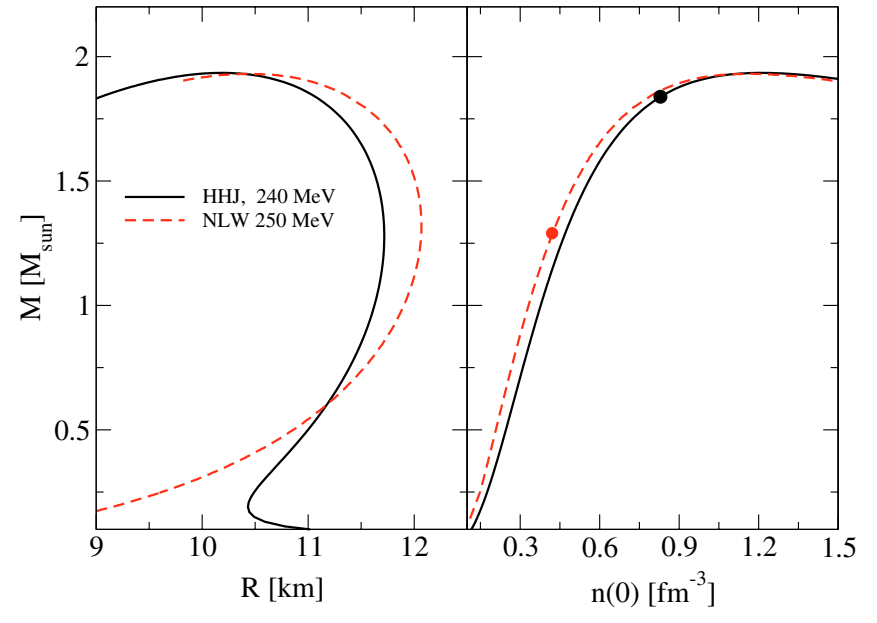

Fig. 2. The relation of gravitational mass to radius and of mass to central density for NS configurations corresponding to the HHJ (solid lines) and the NLW (dashed lines) model EoS. Dots indicate the DU threshold. The possibility of pion condensation is suppressed.

Figure 2 (left panel) shows the mass-radius relation and (right panel) the mass-central density relation. Solid lines are for the HHJ EoS. To check an alternative possibility and to demonstrate the sensitivity of the value of the DU threshold density to the selected model we also use a version of the relativistic non-linear Walecka (NLW) model in the parameterization of Kolomeitsev \& Voskresensky (2003). The parameters of the NLW model are adjusted to the following bulk parameters of the nuclear matter at saturation: $n_{0}=0.16 \mathrm{fm}^{-3}$, binding energy $E_{\text {bind }}=-15.8 \mathrm{MeV}$, compression modulus $K=250 \mathrm{MeV}$, symmetry energy $a_{\text {sym }}=28 \mathrm{MeV}$, and effective nucleon mass $m_{\mathrm{N}}^{*}\left(n_{0}\right)=0.8 m_{\mathrm{N}}$. The corresponding coupling constants are as follows:

$$
\begin{aligned}
\frac{g_{\omega \mathrm{N}}^{2} m_{\mathrm{N}}^{2}}{m_{\omega}^{2}} & =91.2506 \\
\frac{g_{\sigma \mathrm{N}}^{2} m_{\mathrm{N}}^{2}}{m_{\sigma}^{2}} & =195.597 \\
\frac{g_{\rho \mathrm{N}}^{2} m_{\mathrm{N}}^{2}}{m_{\rho}^{2}} & =77.4993 \\
b & =0.00867497 \\
c & =0.00805981
\end{aligned}
$$

(note that we corrected obvious misprints in $b$ and $c$ in the paper of Kolomeitsev \& Voskresensky 2003). This model was constructed in such a way that it almost reproduces the thermodynamic properties in the model of HHJ. However, not having enough free parameters, all NLW-based models yield significantly lower threshold densities for the DU process than those given by variational calculations. Migdal et al. (1990) treated this fact as a weak point of the relativistic mean field models. Therefore they disregarded the DU process in their subsequent analysis, concentrating on the pion softening and the PU possibility. Lattimer et al. (1991) used this to develop the DU-based scenario for the NS cooling. Anyhow, in the given NLW model the threshold density for the DU process is $n_{\mathrm{c}}^{\mathrm{DU}} \simeq 2.62 n_{0}$ $\left(M_{\mathrm{c}}^{\mathrm{DU}} \simeq 1.29 M_{\odot}\right)$. The dots in Fig. 2 (right panel) indicate the 


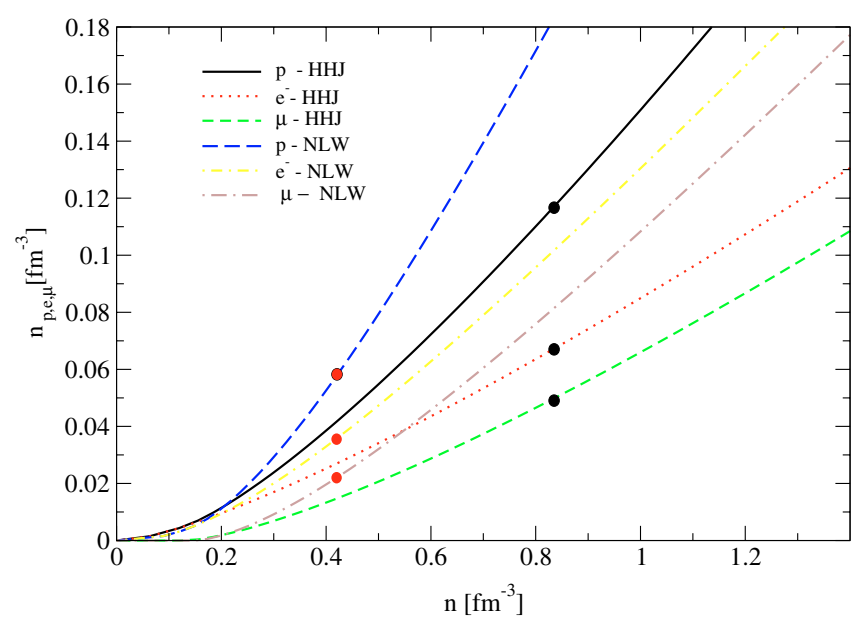

Fig. 3. The densities of charged particles as a function of the baryon density for the HHJ and the NLW model EoS. The threshold densities for the DU process are indicated by dots. The influence of pion condensation for $n>n_{\mathrm{c}}^{\mathrm{PU}}$ is neglected.

threshold densities for the DU process. The influence of pion condensation on the EoS for $n>n_{\mathrm{c}}^{\mathrm{PU}}$ is assumed to be small and is therefore suppressed. For the HHJ EoS the threshold density $n_{\mathrm{c}}^{\mathrm{PU}}=3 n_{0}$ corresponds to a NS mass $M_{\mathrm{c}}^{\mathrm{PU}} \simeq 1.32 M_{\odot}$. From Fig. 2 one can see that deviations in the $M(n)$ relation for HHJ and NLW EoS are minor, whereas the DU thresholds are quite distinct.

Figure 3 demonstrates the concentrations of $\mathrm{p}$, e and $\mu^{-}$as a function of the baryon density for the HHJ and NLW models, respectively. All these dependencies are quite different for these two models. This means that relativistic mean field models give good descriptions of the thermodynamic properties but yield a quite different cooling picture compared to that given by the more microscopically based variational calculations of Argonne-Urbana. The possibility of charged pion condensation is suppressed here. Otherwise for $n>n_{\mathrm{c}}^{\mathrm{PU}}$ the isotopic composition might change (Migdal et al. 1990) in favor of an increase of the proton fraction and a smaller critical density for the DU reaction.

\subsection{NS crust}

The density $n \sim 0.5-0.7 n_{0}$ is the boundary between the NS interior and the inner crust. The latter is constructed of a pasta phase discussed by Ravenhall et al. (1983), (see the recent work of Maruyama et al. 2004). Then there is the outer crust and the envelope. Note that our code generates a temperature profile that is inhomogeneous during the first $10^{2}-10^{3} \mathrm{yr}$. The influence of the crust on the cooling and heat transport is rather minor, basically because of its rather low mass content. Therefore the temperature changes only slightly in the region between the crust and the envelope.

\subsection{Envelope}

Further on we need a relation between the crust and the surface temperature for NS. A sharp change of the temperature

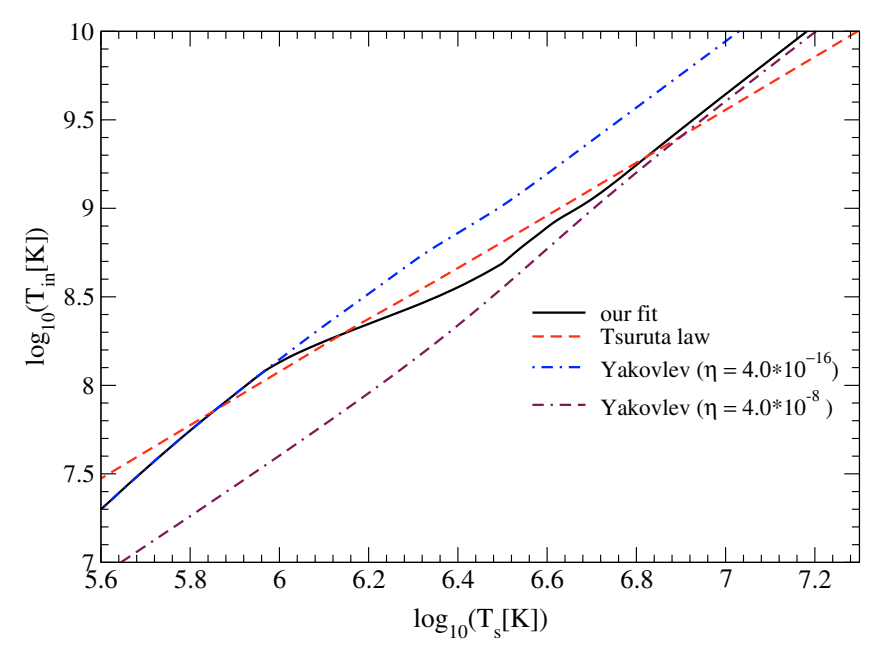

Fig. 4. The relation between the inner temperature and the surface temperature for different models used in our calculations. Dash-dotted curves indicate boundaries of possible $T_{\mathrm{s}}=f\left(T_{\mathrm{in}}\right.$ ) relations (Yakovlev et al. 2004b).

occurs in the envelope. This $T_{\mathrm{s}}-T_{\text {in }}$ relation has been calculated in several studies (Glen \& Sutherland 1980; Yakovlev et al. 2004b) depending on the assumed value of the magnetic field at the surface and some uncertainties in our knowledge of the structure of the envelope. Figure 4 shows a range of available $T_{\mathrm{s}}=f\left(T_{\mathrm{in}}\right)$ curves, taken from Yakovlev et al. (2004b). The solid curve, $T_{\mathrm{s}}=T_{\mathrm{s}}^{\mathrm{fit}}=f\left(T_{\mathrm{in}}\right)$, corresponds to our best fit of the "slow cooling" objects. It matches the upper boundary (the dash-dotted curve for $\eta=4.0 \times 10^{-16}$ ) for rather low $T_{\mathrm{s}}$ (in the interval $\log T_{\mathrm{s}}[K] \simeq 5.8-6$ ) and the lower boundary (the dashdotted curve for $\left.\eta=4.0 \times 10^{-8}\right)$ for high $T_{\mathrm{s}}\left(\log T_{\mathrm{s}}[K] \geq 6.8\right)$. The dashed curve shows the so called simplified Tsuruta law $T_{\mathrm{s}}^{\text {Tsur }}=\left(10 T_{\mathrm{in}}\right)^{2 / 3}$ used in many old cooling calculations. Further we will vary different possibilities. The dependence of the results on the $T_{\mathrm{s}}-T_{\text {in }}$ relation is demonstrated below in Figs. 9, 10, 12-16.

\section{Main cooling regulators}

We compute the NS thermal evolution adopting our fully general relativistic evolutionary code. This code was originally constructed for the description of hybrid stars by Blaschke et al. (2001). The main cooling regulators are the thermal conductivity, the heat capacity and the emissivity. To ensure that our results are comparable with those of other groups we try to be as close as possible to their inputs for those quantities that we did not calculate ourselves. Then we add inevitable changes in order to improve the EoS and to include medium effects.

\subsection{Thermal conductivity}

We take the electron-electron contribution to the thermal conductivity and the electron-proton contribution for normal protons from Gnedin \& Yakovlev (1995). The total contribution related to electrons is then given by

$1 / \kappa_{\mathrm{e}}=1 / \kappa_{\mathrm{ee}}+1 / \kappa_{\mathrm{ep}}$. 
For $T>T_{\text {cp }}$ (normal "n" matter), we have $\kappa_{\mathrm{ep}}^{\mathrm{n}}=\kappa_{\mathrm{ep}}$. For $T<T_{\text {cp }}$ (superfluid "s" matter), Gnedin \& Yakovlev (1995) suggested to drop the superfluid contribution $1 / \kappa_{\mathrm{ep}}^{\mathrm{s}}$. We use the expression

$\kappa_{\mathrm{ep}}^{\mathrm{s}}=\kappa_{\mathrm{ep}} / \xi_{\mathrm{p}}>\kappa_{\mathrm{ep}}^{\mathrm{n}}$,

that gives a crossover from the non-superfluid case to the superfluid case. The vanishing of $\kappa_{\mathrm{ep}}^{\mathrm{s}}$ for $T \ll T_{\mathrm{cp}}$ is a consequence of the scattering of superfluid protons on the electron impurities, see Blaschke et al. (2001). Following Eq. (7) we get $\kappa_{\mathrm{e}}^{\mathrm{n}}<$ $\kappa_{\mathrm{e}}^{\mathrm{s}}$. Surprisingly, this is in disagreement with Fig. 4 of Baiko et al. (2001), where probably the curves "SF1" and "SF0" should be interchanged.

For the nucleon contribution,

$1 / \kappa_{\mathrm{n}}=1 / \kappa_{\mathrm{nn}}+1 / \kappa_{\mathrm{np}}$,

we use the result of Baiko et al. (2001) that includes corrections for the superfluidity. Although some medium effects are incorporated in this work, the nucleon-hole corrections of correlation terms and the modification of the tensor force are not included. This should modify the result. However, since we did not calculate $\kappa_{\mathrm{n}}$ ourselves, we may only roughly estimate the modification. As we have shown above in Fig. 1, not too close to the critical point of the pion condensation the squared matrix element of the $N N$ interaction $|M|_{\text {med }}^{2} \sim p_{F, n}^{2} \Gamma^{2} / \widetilde{\omega}^{2}$ is of the order of the corresponding quantity $|M|_{\mathrm{vac}}^{2} \sim p_{F, n}^{2} /\left[m_{\pi}^{2}+p_{F, n}^{2}\right]$ estimated with the free one pion exchange, whereas $|M|_{\text {med }}^{2}$ may significantly increase for $n \sim n_{\mathrm{c}}^{\mathrm{PU}}$. To simulate the effect we just allow for the variation of $\kappa_{\mathrm{n}}$ multiplying it by the factors $\zeta_{\kappa}=10$ (fast transport) and $\zeta_{\kappa}=0.3$ (slow transport), see Figs. 7, 17 below. The former case is relevant for rather massive stars, and the latter for rather light stars. A suppression of the nucleonnucleon amplitude compared to the one for the free one pion exchange model for $n \lesssim n_{0}$ is motivated by the in-medium $T$ matrix calculations by Blaschke et al. (1995).

The total thermal conductivity is the straight sum of the partial contributions

$\kappa_{\mathrm{tot}}=\kappa_{\mathrm{e}}+\kappa_{\mathrm{n}}+\ldots$

Other contributions to this sum are smaller than those presented explicitly $\left(\kappa_{\mathrm{e}}\right.$ and $\left.\kappa_{\mathrm{n}}\right)$.

\subsection{Heat capacity}

The heat capacity contains nucleon, electron, photon, phonon, and other contributions. The main in-medium modification of the nucleon heat capacity is due to the density dependence of the effective nucleon mass. We use the same expressions as Schaab et al. (1997). The main regulators are the nucleon and the electron contributions. For the nucleons $(i=\mathrm{n}, \mathrm{p})$, the specific heat is (Maxwell (1979))

$c_{i} \sim 10^{20}\left(m_{i}^{*} / m_{i}\right)\left(n_{i} / n_{0}\right)^{1 / 3} \zeta_{i i} T_{9} \operatorname{erg~cm}^{-3} \mathrm{~K}^{-1}$,

for the electrons it is

$c_{\mathrm{e}} \sim 6 \times 10^{19}\left(n_{\mathrm{e}} / n_{0}\right)^{2 / 3} T_{9} \mathrm{erg} \mathrm{cm}^{-3} \mathrm{~K}^{-1}$.
Near the phase transition point the heat capacity acquires a fluctuation contribution. For the first order pion condensation phase transition this additional contribution contains no singularity, different from what is expected for a second order phase transition (Voskresensky \& Mishustin; Migdal et al. 1990). Finally, the nucleon contribution to the heat capacity may increase by up to several times in the vicinity of the pion condensation point. The effect of this correction on global cooling properties is rather unimportant.

The symmetry of the $3 \mathrm{P}_{2}$ superfluid phase allows the contribution of Goldstone bosons (phonons):

$C_{G} \simeq 6 \times 10^{14} T_{9}^{3} \frac{\mathrm{erg}}{\mathrm{cm}^{3} \mathrm{~K}}$,

for $T<T_{\text {cn }}\left(3 \mathrm{P}_{2}\right), n>n_{\text {cn }}\left(3 \mathrm{P}_{2}\right)$. We also include this contribution in our study although its effect on the cooling is rather minor.

\subsection{Emissivity}

We adopt the same set of partial emissivities as in the work of Schaab et al. (1997). The phonon contribution to the emissivity of the $3 \mathrm{P}_{2}$ superfluid phase is negligible. The main emissivity regulators are the MMU, see the above rough estimate (5), the $n \mathrm{PBF}$ and the $p \mathrm{PBF}$ processes, see the above rough estimate (4).

Only the qualitative behavior of the interaction shown in Fig. 1 is motivated by a microscopic analysis whereas the actual numerical values of the correlation parameter and the pion gap are rather uncertain. Therefore we vary the values $\Gamma(n)$ and $\omega^{* 2}(n)$ in accordance with the above discussion of Fig. 1. By this we check the relevance of alternative possibilities: a) no pion condensation and a saturation of the pion softening with increasing density, b) presence of pion condensation.

We also add the contribution of the DU process for $n>n_{\mathrm{c}}^{\mathrm{DU}}$, see the above rough estimate (3).

All emissivities are corrected for correlation effects. The PU process contains an extra $\Gamma_{\mathrm{s}}^{2}$ factor compared to the DU process. Further suppression of the PU emissivity comes from the fact that it is proportional to the squared pion condensate mean field $|\varphi|^{2}$. Near the critical point $|\varphi|^{2} \sim 0.1$ increases with density up to $|\varphi|^{2} \sim f_{\pi}^{2} / 2$, where $f_{\pi} \simeq 93 \mathrm{MeV}$ is the pion decay constant. Finally, the PU emissivity is about 1-2 orders of magnitude suppressed compared to the DU one. Moreover, we adopt the same gap dependence for the PU process as for the DU process.

\subsection{Nucleon superfluidity}

In spite of many calculations, values of nucleon gaps in dense NS matter are poorly known. This is the consequence of the exponential dependence of the gaps on the poorly known NN interaction for $n \neq n_{0}$. Recent calculations of Schwenk \& Friman (2004) who included medium effects in the evaluation of the $3 \mathrm{P}_{2}$ gap demonstrate its strong suppression down to values $\lesssim 10 \mathrm{keV}$. Together with previous findings of Schulze et al. (1996) and Lombardo \& Schulze (2001) they lead us to consider the possibility of rather suppressed gaps. 


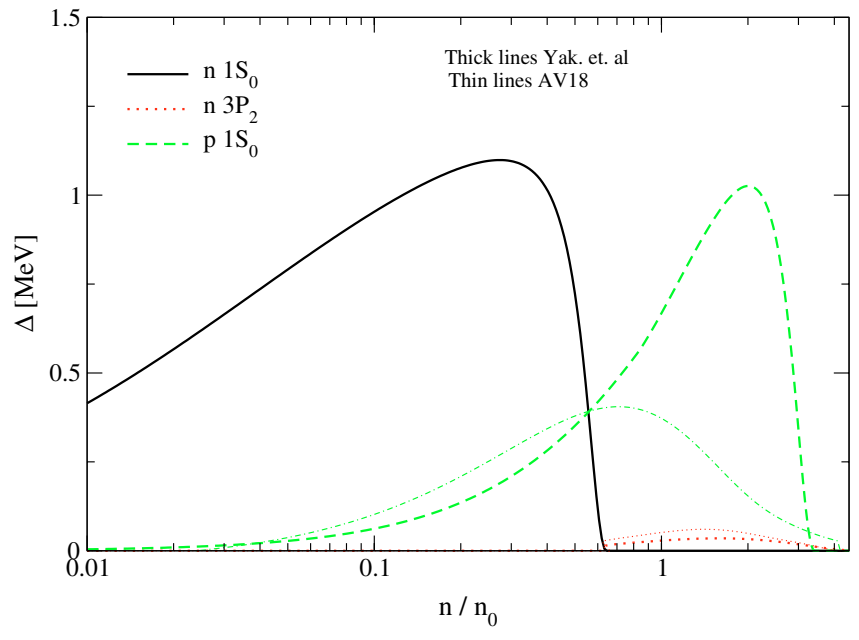

Fig. 5. Neutron and proton pairing gaps according to Yakovlev et al. (2004a) (thick solid, dashed and dotted lines) and to Takatsuka \& Tamagaki (2004) (thin lines).

Below we start with the model used by Yakovlev et al. (2004a). Also we use the gaps from the recent work of Takatsuka \& Tamagaki (2004) to compare the results. The gaps are presented in Fig. 5. Thick lines stand for the gaps used by Yakovlev et al. (2004a) and thin lines for those of Takatsuka \& Tamagaki (2004) who used the AV18 interaction model by Wiringa et al. (1995). The $1 \mathrm{~S}_{0}$ neutron gap is taken to be the same in both models. We vary the gaps within wide limits to check the sensitivity of the results to their values. As we will see below the cooling curves are sensitive to the magnitudes and to the density dependence of the gaps. Therefore further microscopic studies of the gaps are required.

\subsection{KDU, HDU, QDU and other}

The phase structure of dense NS matter might be very rich, including $\pi^{0}, \pi^{ \pm}$condensates and $\bar{K}^{0}, K^{-}$condensates in both $\mathrm{S}$ and $\mathrm{P}$ waves (Kolomeitsev \& Voskresensky 2003), charged $\rho$-meson condensation (Voskresensky 1997), coupling of condensates (Umeda et al. 1994), fermion condensation yielding an efficient DU-like process in the vicinity of the pion condensation point (with the emissivity $\varepsilon_{v} \sim 10^{27} T_{9}^{5}, m_{\mathrm{N}}^{*} \propto 1 / T$, see Voskresensky et al. 2000), hyperonization (see Takatsuka \& Tamagaki 2004), quark matter with different phases such as the so called 2SC, CFL, CSL phases, plus their interaction with meson condensates (Rajagopal \& Wilczek 2000; Blaschke et al. 2001, and references therein), and different mixed phases. In the present work, we suppress all these possibilities of extra efficient cooling channels. They are effectively simulated by our PU choice. The quark matter effects need a special discussion. We will return to the latter possibility in a subsequent publication.

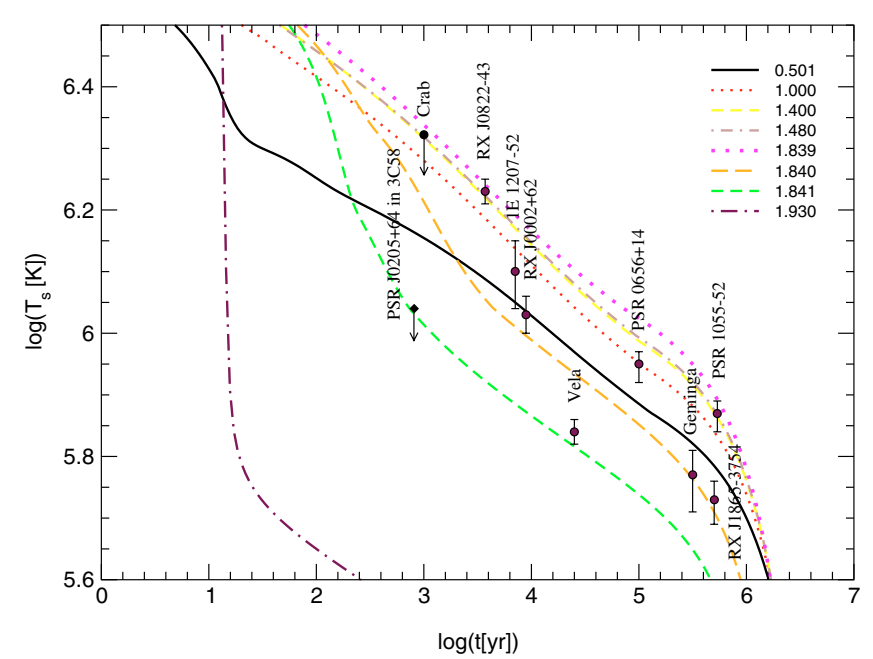

Fig. 6. Cooling of HHJ configurations without medium effects and without PU for different masses of NS, $T_{\mathrm{s}}-T_{\text {in }}$ relation according to our fit.

\section{Numerical results}

\subsection{Cooling of normal NS}

Although we have no doubts about the presence of nucleon superfluidity in NS interiors, we consider first the case of its complete absence. The reasons for this are the following: i) In this way we compare our results with previous calculations. ii) With this example we select more essential and less essential ingredients in this many-parameter problem. The results are more transparent if they are demonstrated for a simplified example since the general case introduces new uncertain parameters and is more involved. iii) The actual values of gaps might be essentially smaller than those estimated in the literature (Lombardo \& Schulze 2001; Schwenk \& Friman 2004). This is because most calculations did not include a proper medium dependence of the NN interactions. Thus we discuss the limiting case of largely suppressed gaps.

Figure 6 demonstrates the cooling evolution (for $T_{\mathrm{s}}^{\mathrm{fit}}(t)$ dependence) of a normal NS for the HHJ EoS. Medium effects and the PU possibility are disregarded (Standard scenario). We see that one can easily explain the slow cooling points. Curves for NS masses in the range $M \simeq(1-1.839) M_{\odot}$ lie in this region. For $M \simeq 1.839 M_{\odot}$ the efficient DU process is switched on within the HHJ EoS ("Standard + exotics DU" scenario). Therefore, the curve corresponding to $M \simeq 1.841 M_{\odot}$ drops steeply and this explains the rapid cooling points. The intermediate cooling points can be explained either by a very low NS mass (see the curve for $M \simeq 0.5 M_{\odot}$ ), or by $M \simeq 1.840 M_{\odot}$. However, it seems rather unrealistic that 4 out of 10 objects either relate to very low masses like $M \simeq 0.5 M_{\odot}$, or are highly massive, $M \simeq 1.840 M_{\odot}$, where the DU process is switched on. Rapid cooling points are explained by very massive objects $\left(M \simeq 1.841 M_{\odot}\right)$ with the DU process. They could also be explained by very low-mass objects $\left(M \sim 0.1 M_{\odot}\right)$. We drop the latter possibility as a rather unrealistic one and consider $M \geq 0.5 M_{\odot}$. We notice that one needs to introduce some 


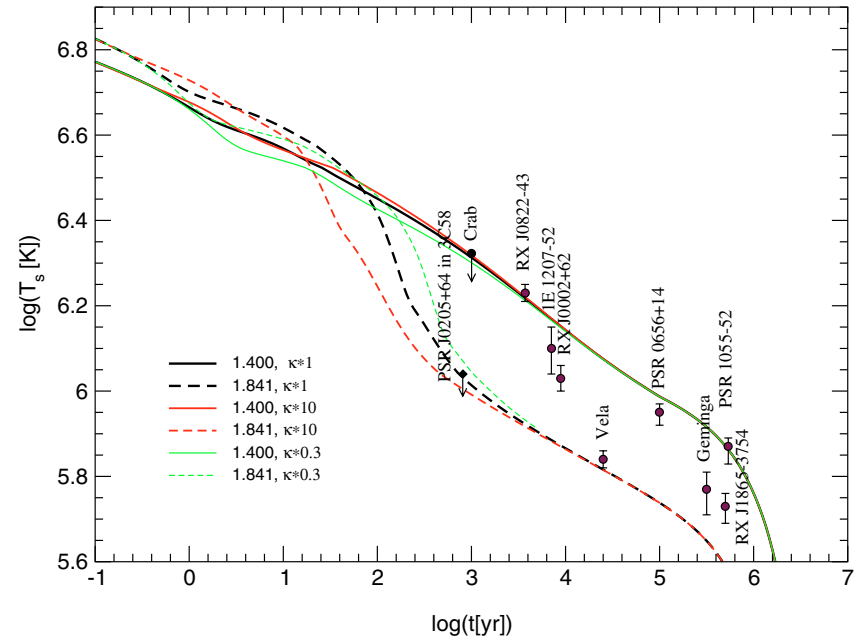

Fig. 7. The influence of the heat conductivity on the scenario of Fig. 6. Results for two representative configurations with masses $M=1.40 M_{\odot}$ (solid lines) and $M=1.841 M_{\odot}$ (dashed lines) are shown. We vary the heat conductivity by factors $\zeta=10$ and $\zeta=0.3$.

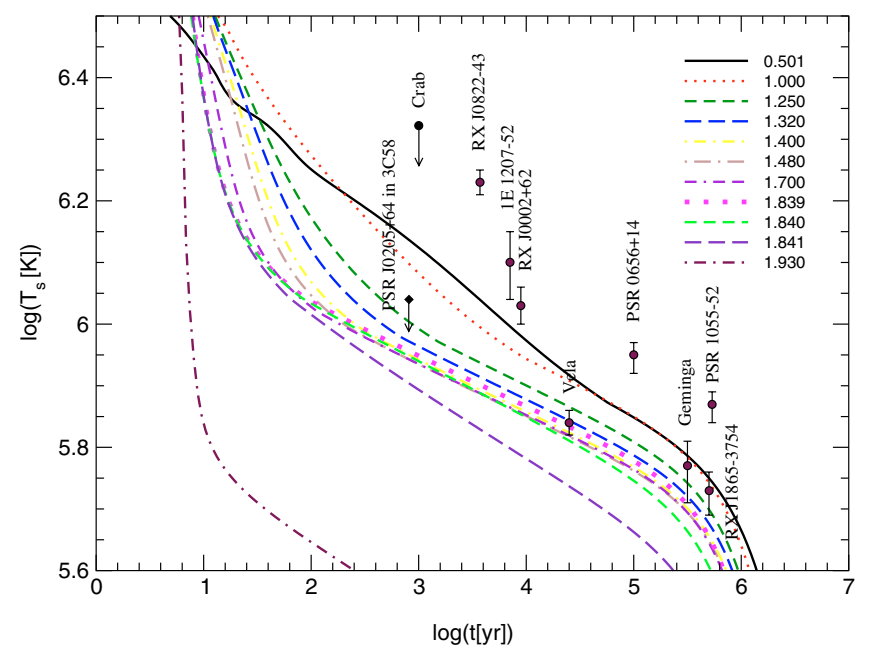

Fig. 8. Cooling evolution of NS with normal matter HHJ EoS (for $T_{\mathrm{s}}^{\mathrm{fit}}$ ) including the medium modification of $\mathrm{MU}$ and other processes (MMU, MNB, etc.), without pion condensation.

non-standard mechanisms to produce NS with masses below $1 M_{\odot}($ Popov 2004).

Thus the picture as a whole looks unsatisfactory. Only slow cooling data are appropriately explained in a reasonable NS mass interval of 1.0-1.839 $M_{\odot}$. The explanation of intermediate cooling and rapid cooling, although possible, needs very unnatural assumptions.

Figure 7 demonstrates the sensitivity of the cooling curves to the variation of $\kappa_{\mathrm{n}}$. We scale $\kappa_{\mathrm{n}}$ by factors $\zeta=10$ and $\zeta=0.3$. The former case is meaningful for heavy objects and the latter for low-mass objects. We see that neither increasing nor decreasing the thermal conductivity changes the picture as a whole, or the conclusion drawn above. Transport is relevant only up to the first $10^{3} \mathrm{yr}$ when the details of the $T_{\mathrm{s}}-T_{\text {in }}$ relation as well as the temperature and density dependences of the cooling regulators determine the response of the cooling curves to a rescaling of the heat conductivity by the factor $\zeta$.



Fig. 9. Same as Fig. 8, including pion condensation for $n>3 n_{0}$.

Figure 8 allows medium effects the strength of which is assumed to be saturated with increasing density (no $\pi$ condensation for $n>3 n_{0}$ ). Thus we use curves $1 \mathrm{a}, 1 \mathrm{~b}$ of Fig. 1 . We see that the inclusion of medium effects into the calculation of the emissivity (MMU, MNB and others) significantly decreases all curves. This allows us to explain reasonably well the rapid cooling points but the slow cooling and the intermediate cooling cannot be addressed without the inclusion of superfluidity. A partial suppression of medium effects by scaling $\omega^{*}$ does not improve the picture.

Comparison of Figs. 8 and 6 shows that the inclusion of medium effects regulates the mass dependence of the curves. In Fig. 6 the curves rise with increasing NS mass (for objects with $M \leq 1.839 M_{\odot}$, below the DU threshold) whereas in Fig. 8 the trend is changed to the opposite.

Figure 9 also allows for $\pi$ condensation for $n>3 n_{0}(M>$ $1.32 M_{\odot}$ for the HHJ EoS). Now we use the curves 1a, 2, 3 of Fig. 1. The picture remains almost the same as that shown in Fig. 8. However, objects with $M \geq 1.32 M_{\odot}$ are cooling still faster by the efficient PU reaction. If we would assume $n_{\mathrm{c}}^{\mathrm{PU}} \simeq 2.5 n_{0}$ then the pion condensation would already start for NS masses $M>1.08 M_{\odot}$.

Figure 10 demonstrates the same cooling evolution of normal NS as Fig. 9, but for the Tsuruta relation $T_{\mathrm{s}}^{\mathrm{Tsur}}\left(T_{\mathrm{in}}\right)$. One can explain the rapid cooling and the intermediate cooling but cannot explain the slow cooling. We have checked that the use of other $T_{\mathrm{s}}-T_{\text {in }}$ relations within the band given by Fig. 4 does not change the general trends. Medium effects and the possibility of $\pi$ condensation, although they regulate the NS massdependence show too rapid cooling. We see that without superfluidity the picture is unsatisfactory. Therefore we conclude that the cooling data require nucleon superfluidity.

\subsection{Cooling of superfluid NS}

First we check the best fit of the model by Kaminker et al. (2002) and Yakovlev et al. (2004a), where gaps are given in Fig. 5 (thick lines). In order to get a fit of the data within their "Standard $+D U$ " scenario Kaminker et al. (2002) and 


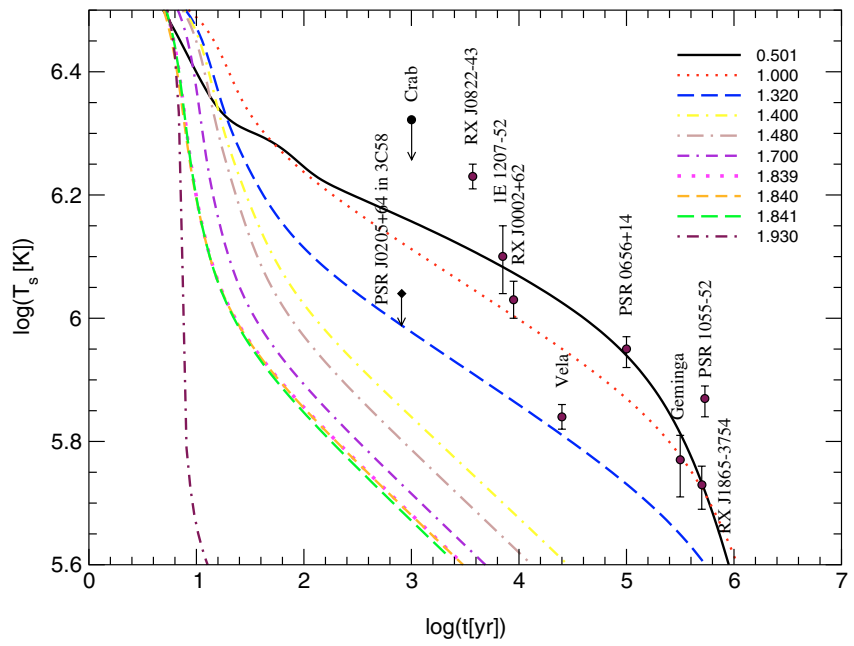

Fig. 10. Same as Fig. 9, but with a $T_{\mathrm{s}}-T_{\text {in }}$ relation given by the Tsuruta law.



Fig. 11. Cooling of NS configurations with superfluid nuclear matter without medium effects and pion condensation. The gaps are taken as in Yakovlev et al. (2004a), see Fig. 5, the neutron $3 \mathrm{P}_{2}$ gap is additionally suppressed by a factor 10 , the $T_{\mathrm{s}}-T_{\text {in }}$ relation is given by $T_{\mathrm{s}}^{\mathrm{fit}}(t)$.

Yakovlev et al. (2004a) were forced to additionally switch off the $3 \mathrm{P}_{2}$ neutron gap. Since the full switching off of the $3 \mathrm{P}_{2}$ gap is not supported by microscopic calculations we simulate the same effect by introducing a scaling factor 0.1 for the $3 \mathrm{P}_{2}$ neutron gap, shown in Fig. 5 by a corresponding solid line. Then the magnitude of the $3 \mathrm{P}_{2}$ gap becomes comparable with the value following from the findings of Schwenk \& Friman (2004), who estimated the $3 \mathrm{P}_{2}$ neutron gap including medium effects.

Figure 11 demonstrates the cooling evolution $\left(T_{\mathrm{s}}^{\mathrm{fit}}(t)\right.$ dependence) of the above choice of gaps for a NS with HHJ EoS. Medium effects are not included. The curves lie higher than those in Fig. 6. We see that for $M \leq 1.839 M_{\odot}$ when the DU process is switched off, all the curves show too slow cooling even not explaining the slow cooling data points (for $M \gtrsim$ $\left.1 M_{\odot}\right)$. We also checked the artificially suppressed rate of the $p \mathrm{PBF}$ process used by Kaminker et al. (2002) and Yakovlev et al. (2004a). Suppression of the rate by a factor of 10 does



Fig. 12. Same as in Fig. 11 including medium effects without pion condensation (curves 1a, 1b in Fig. 1).

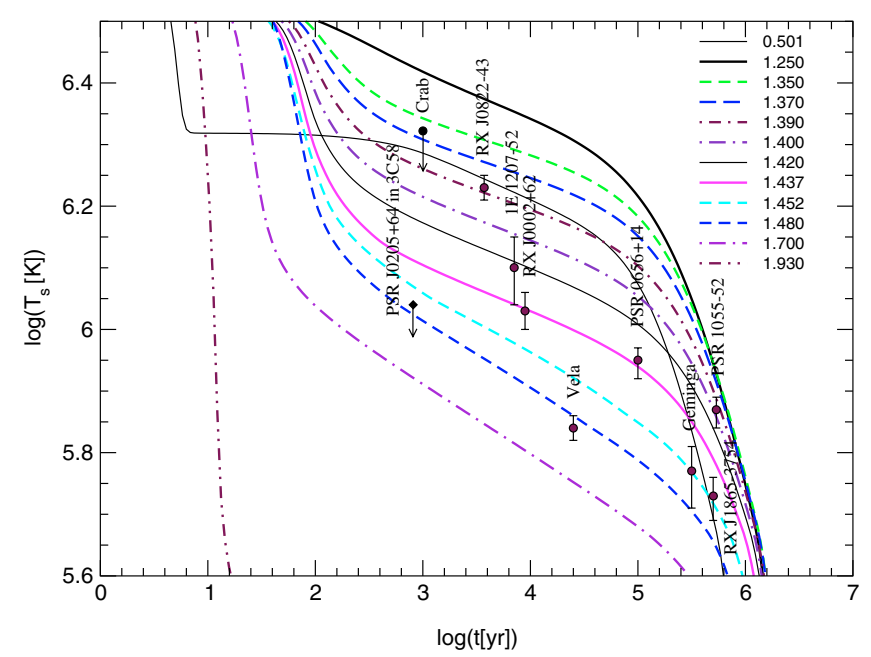

Fig. 13. Same as in Fig. 12 but using the Tsuruta law.

not significantly affect the curves and does not change the conclusion. The general trends of the curves are similar to those of Kaminker et al. (2002). However, within the HHJ EoS the intermediate cooling points and the rapid cooling points can be explained only by objects with $M>1.839 M_{\odot}$ and DU cooling which seems quite unsatisfactory to us. Comparison of Fig. 11 with Fig. 6, computed within the same scenario but without inclusion of the superfluidity, shows that both choices suffer from the same shortcomings.

Figure 12 allows for medium effects (we use curves $1 \mathrm{a}, 1 \mathrm{~b}$ of Fig. 1, no $\pi$ condensation for $n>3 n_{0}$ ). All the data are explained by masses in the interval $M \simeq 1.39-1.84 M_{\odot}$. The regular behavior is clearly seen. More massive objects cool faster than less massive ones. The star with mass $1.25 M_{\odot}$ may belong to the old and hot objects like PSR 1055-52.

We have checked the sensitivity of the result to the $T_{\mathrm{s}}-T_{\text {in }}$ relation. Figure 13 uses the Tsuruta law whereas Fig. 14 probes the choice of Yakovlev et al. (2004a) for $\eta=4.0 \times 10^{-16}$. We see that a variation of the $T_{\mathrm{s}}-T_{\text {in }}$ relation does not change the picture as a whole. Only the interval of masses that cover the 


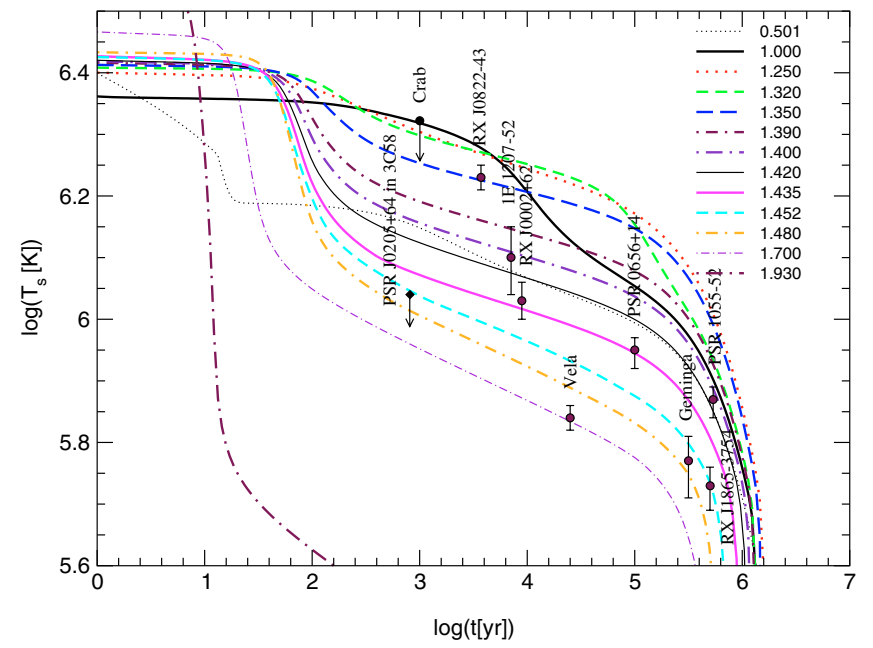

Fig. 14. Same as in Fig. 12 but $T_{\mathrm{s}}-T_{\text {in }}$ relation according to Yakovlev et al. (2004a), $\eta=4 \times 10^{-16}$.



Fig. 15. Same as in Fig. 12 including $\pi$ condensation.

data is slightly changed. This interval is $M \simeq 1.36-1.50 M_{\odot}$ according to Fig. 13 and $M \simeq 1-1.75 M_{\odot}$ according to Fig. 14 .

Figure 15 allows for $\pi$ condensation for $n>3 n_{0}$. Now we use curves 1a, 2, 3 of Fig. 1 . The $T_{\mathrm{s}}-T_{\text {in }}$ relation is according to our fit. The mass interval that covers the data is $M \simeq 1.37-1.46 M_{\odot}$.

Figure 16 shows the same as Fig. 15 but for the Tsuruta law. In both cases the picture remains almost the same as that shown in Figs. 13 and 12, respectively. The difference starts for masses $\geq 1.32 M_{\odot}$ when the efficient PU process is switched on. The interval of masses that covers the data is $M \simeq 1.36-1.452 M_{\odot}$. Using other $T_{\mathrm{s}}-T_{\text {in }}$ dependences does not change the picture. Thus we may conclude that the $\pi$ condensation does not contradict the data but, on the other hand, this scenario is not motivated by the existing cooling data. The hyperon enhanced cooling or kaon condensation cooling do not change the picture if their critical densities are $\gtrsim 3 n_{0}$. Note that the transition density cannot be very low. At least we should have $n_{\mathrm{c}}^{\mathrm{PU}} \geq 2.5-2.7 n_{0}$ for the charged pion condensation for the given EoS. The neutral PU process might be additionally suppressed compared to the charged one (Voskresensky 2001)

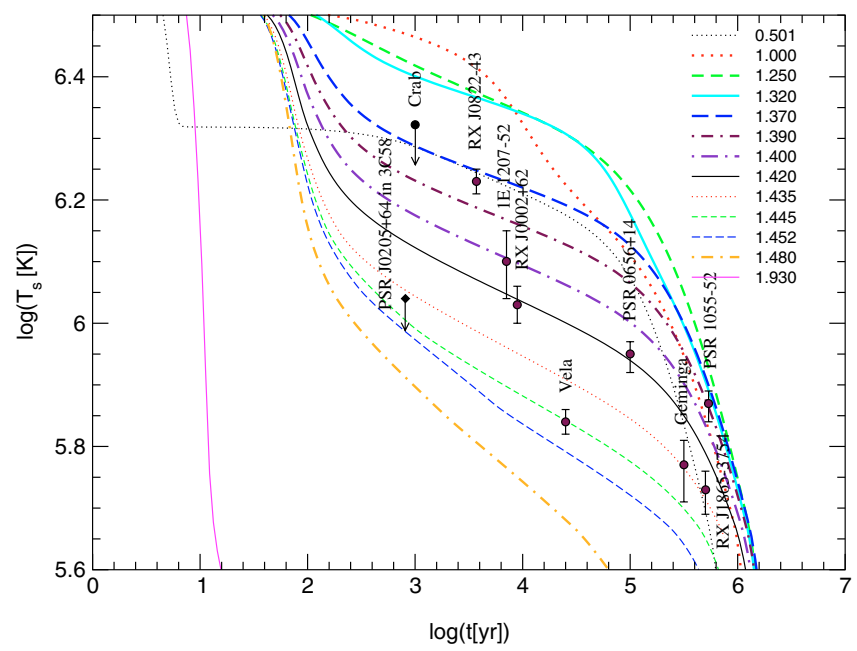

Fig. 16. Same as in Fig. 15 using the Tsuruta law.

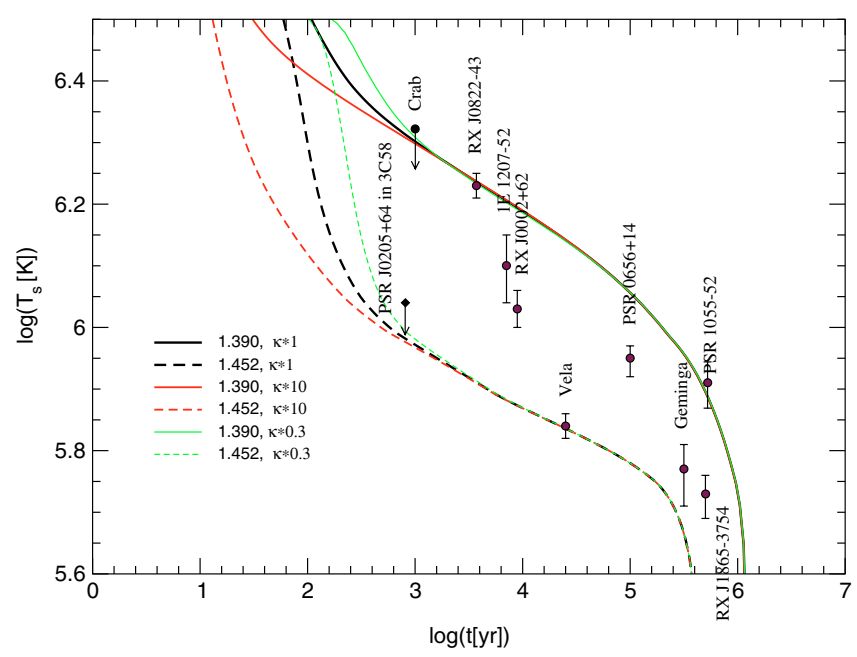

Fig. 17. The influence of a change of the heat conductivity on the scenario of Fig. 15.

which may weaken the above restriction. Otherwise we would get too fast cooling already for low mass objects and a NS with $M \simeq 1.4 M_{\odot}$ would cool faster than is compatible with modern data.

Figure 17 demonstrates the sensitivity of the curves presented in Fig. 15 to the variation of $\kappa_{\mathrm{n}}$. We scale $\kappa_{\mathrm{n}}$ by the factors $\zeta=10$ and $\zeta=0.3$. We see that both increasing and decreasing the thermal conductivity $\kappa_{\mathrm{n}}$ does not change the picture as a whole nor the conclusion drawn above. As in the case when medium effects are suppressed, (Fig. 7), the effect of the transport is relevant for the first $10^{3} \mathrm{yr}$ only.

Figures 18-23 show the dependence of the results on different variations of the gaps, their absolute values and the density dependencies.

We remind the reader that the proton gap was artificially enhanced in Yakovlev et al. (2004a). Figure 18 corresponds to Fig. 15, except for the proton gap which is suppressed by a factor 0.5. A reasonable fit is achieved. In Fig. 19 we further suppress the proton gap (now by a factor 0.2) and we also suppress the $1 \mathrm{~S}_{0}$ neutron gap by a factor 0.5 simulating the medium effects on the gaps. We again obtain a reasonable 


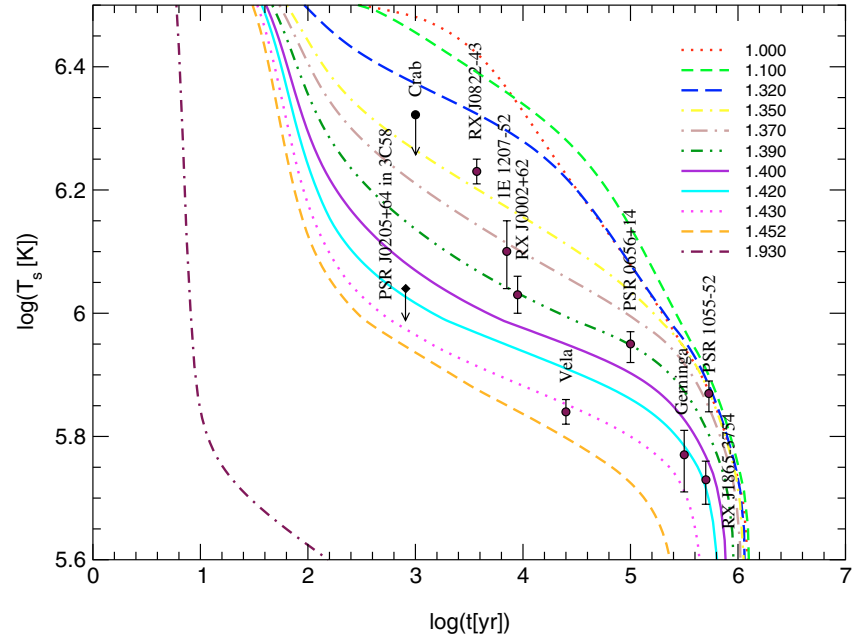

Fig. 18. Same as Fig. 15 but with suppressed proton gap by a factor 0.5 .

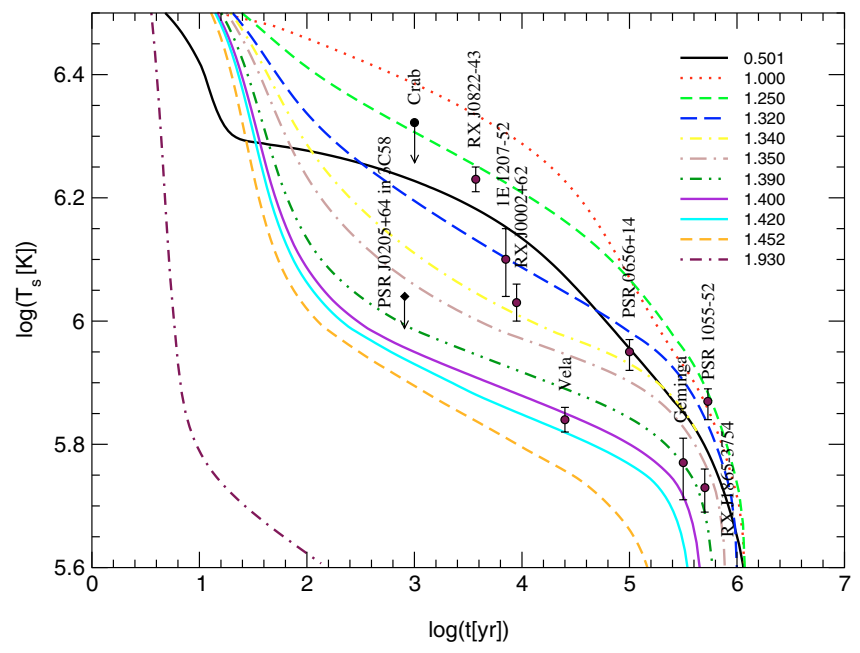

Fig. 19. Same as Fig. 15 but with $1 S_{0}$ neutron gap suppressed by a factor 0.5 and $1 \mathrm{~S}_{0}$ proton gap by a factor 0.2 . The neutron $3 \mathrm{P}_{2}$ gap remains suppressed by 0.1 .

overall fit of the data. The NS masses covering the available data are 1.33-1.44 $M_{\odot}$ for Fig. 18 and 1.23-1.42 $M_{\odot}$ for Fig. 19. However, we have checked that scaling all gaps as shown by the thick lines in Fig. 5 with a factor 0.1 no longer allows a reasonable fit of the data. Thus we have demonstrated the important rôle played by all three types of pairing: $1 S_{0} n n$ and pp pairing as well as $3 \mathrm{P}_{2} \mathrm{nn}$ pairing.

In Fig. 20 we include the same medium effects as in Fig. 12 but we use the gaps according to Takatsuka \& Tamagaki (2004), see thin lines in Fig. 5, additionally suppressing the $3 \mathrm{P}_{2}$ gap by a factor 10 . We see that with the HHJ EoS and the given choice of the gaps we obtain the best fit of the whole set of the data. Thus the pion softening and the appropriate density dependence of the gaps are quite sufficient to explain the modern NS cooling data. The data are covered by NS in the wide mass interval of $0.5-1.84 M_{\odot}$. For masses of $1.0-1.84 M_{\odot}$ the picture is quite regular. Less massive stars cool more slowly, more massive stars cool faster.

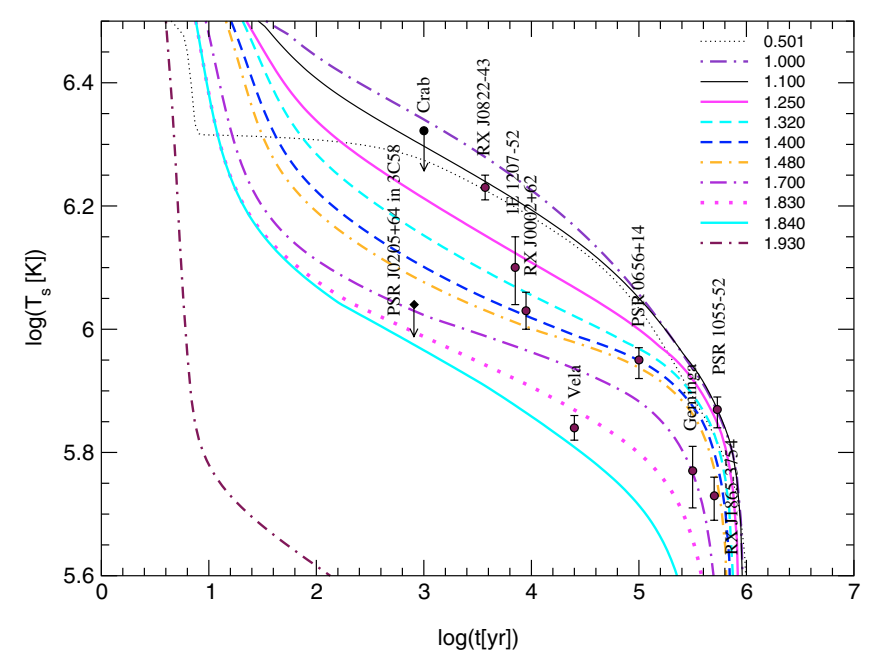

Fig. 20. Same as in Fig. 12 using the gaps of Takatsuka \& Tamagaki (2004), see Fig. 5, with additional suppression of the $3 \mathrm{P}_{2}$ gap by a factor 10 .

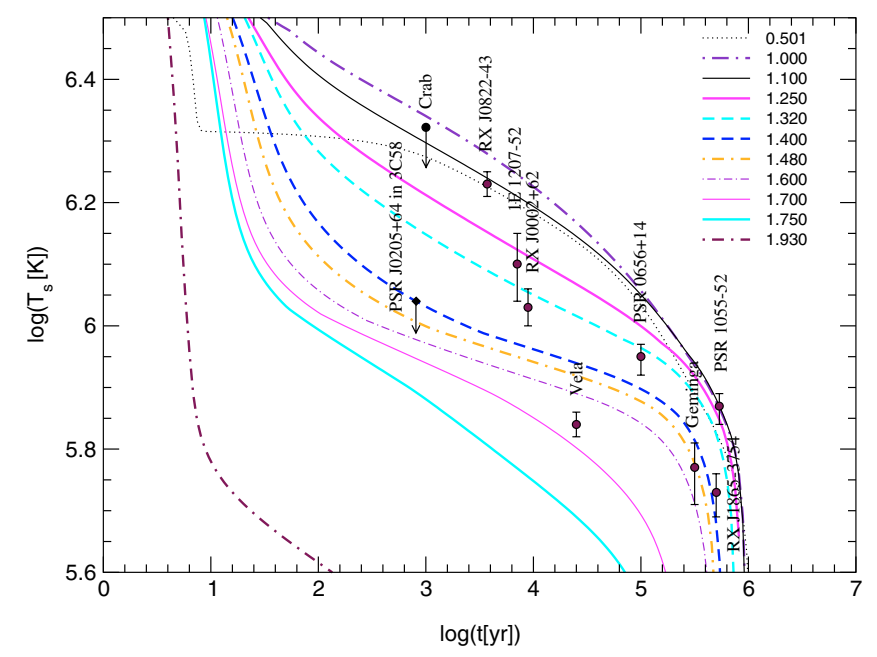

Fig. 21. Same as in Fig. 20 but including pion condensation.

Figure 21 is the same as Fig. 20 but now including the pion condensation. The picture as a whole remains the same. Only the cooling of massive stars is affected which slightly narrows the appropriate NS mass interval from 1.0-1.75 $M_{\odot}$ to $1.0-1.70 M_{\odot}$.

Figures 22 and 23 are the same as Figs. 15 and 21, but now we leave out the suppression factor 0.1 for the $3 \mathrm{P}_{2}$ neutron gap. From Fig. 22 we see that two slow cooling and two intermediate cooling points related to old NS are not explained. In Fig. 23 only two fast cooling data points are explained. We again support the statement that the suppression of the $3 \mathrm{P}_{2}$ gap is indeed motivated by the comparison with the cooling data, at least within the framework of our scenario.

\section{Conclusion}

We have shown that the most up-to-date observed NS cooling data can be well explained. Actually we deal with a manyparameter problem that allows a variation of many quantities. Besides, the data points might be partially shifted from the 


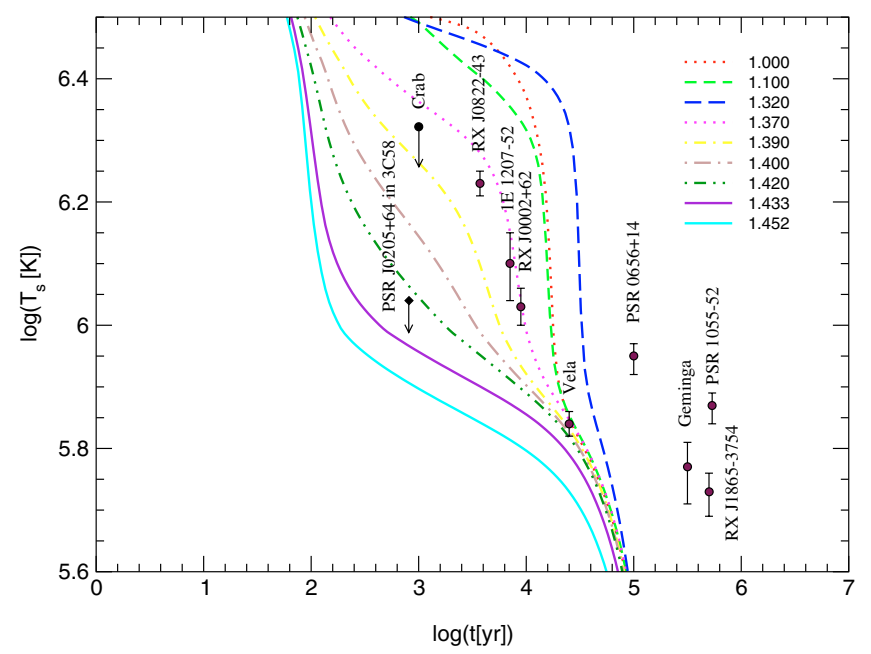

Fig. 22. Same as Fig. 15 without suppression of the $3 \mathrm{P}_{2}$ neutron pairing gap.



Fig. 23. Same as Fig. 21 without suppression of the $3 \mathrm{P}_{2}$ neutron pairing gap.

positions shown in the figures due to a number of uncertainties and assumptions used in their analysis. In the above figures we have illustrated different possibilities, discriminating between more and less probable ones. We have worked out the example of the probably most realistic and microscopically supported EoS which uses the $V 18+\delta v+U I X^{*}$ interaction. We have used the simple parameterization of this EoS suggested by HHJ. In this EoS the DU process does not occur for masses less than $M \simeq 1.839 M_{\odot}$. Thus within this model the Standard $+D U$ scenario would demonstrate that the majority of experimentally measured cooling points relate to very massive NS, with $M \geq 1.84 M_{\odot}$. Such a scenario seems unrealistic.

We exploit in-medium effects in the calculation of the emissivities, the pairing gaps and, less importantly, in the specific heat and the heat conductivity. In general, medium effects result in a significant suppression of the superfluid gaps, especially of the $3 \mathrm{P}_{2} \mathrm{nn}$ gap, and they enhance the cooling rates of the MMU and the MNB processes through the pion softening effect with an increase of the density. Without the latter effect the Standard $+P U$ scenario suffers from an internal inconsistency. Pion condensation cannot take place without preliminary softening of the pion mode at lower densities. A recent argumentation in favor of pion condensation (Akmal et al. 1998; Suzuki et al. 1999) further supports the presence of a precursor pion softening (for details see Migdal et al. 1990). Pion condensation at $n \gtrsim 3 n_{0}$ does not contradict the data but the data can also be explained without pion condensation and other so called "exotics" (KU, HDU, etc.) rather than with pion softening. Based on the works of Schaab et al. (1997), Blaschke et al. (2001) we have further improved our code. We showed that the results are sensitive to the values and the density dependencies of the nucleon superfluid gaps. A strong suppression of the $3 \mathrm{P}_{2}$ gap motivated by theoretical evaluations that incorporate medium effects is indeed required to fit the data. We show that all three groups of points slow cooling, intermediate cooling and rapid cooling are now well explained on the basis of the Nuclear medium cooling scenario demonstrated here, where an important rôle is played by in-medium effects, (cf. Voskresensky 2001).

We may draw the following main conclusions.

i) The normal matter assumption (see Figs. 6-10) seems rather unrealistic, both by itself and in relation to the cooling data. One could explain the data but at the price of ignoring the medium effects in MMU and MNB. Then the intermediate cooling points can only be explained by very low NS masses (as $0.5 M_{\odot}$ ) or, together with rapid cooling points, by the very high NS masses $M>1.839 M_{\odot}$ which allow for the DU process. In the latter case the mass window separating the intermediate cooling and rapid cooling is very narrow. The above price seems too high and we disregard this scenario. Superfluidity is required by the data.

ii) Including superfluid gaps we see, in agreement with recent microscopic findings, that the $3 \mathrm{P}_{2}$ neutron gap should be as small as $\lesssim 10 \mathrm{keV}$. Otherwise one cannot explain several old objects (see Figs. 22, 23). Proton and $1 \mathrm{~S}_{0}$ neutron gaps might also be suppressed by factors of $0.2-0.5$, as it is demonstrated in Figs. 18-21, but the suppression by a factor $\lesssim 0.1$ is no longer permitted.

iii) Inclusion of medium effects associated with pion softening makes it possible to give an appropriate explanation for all the data. As the result of pion softening pion condensation may occur for $n \geq n_{\mathrm{c}}^{\mathrm{PU}}$ ( $n \geq 3 n_{0}$ in our model). Its appearance does not contradict the data (see Fig. 15), but they are fairly well described if the softening effect is rather saturated (see Fig. 12) with increasing density (as demonstrated by curves $1 \mathrm{a}, 1 \mathrm{~b}$ in Fig. 1). At the same time the critical densities for the efficient DU-like exotic processes (such as PU on charged pions) should not be as small as $<2.5 n_{0}$, if the given HHJ EoS is indeed correct. Otherwise one would get too rapid cooling of a NS with the canonical mass of $1.4 M_{\odot}$. This also means that the proper DU threshold density cannot be too low which puts restrictions on the density dependence of the symmetry energy. Both statements might be important in the discussion of heavy ion collision experiments.

iv) We demonstrated a regular mass dependence in the framework of our scenario: for NS masses of $M \gtrsim 1.0 M_{\odot}$ 
less massive NS cool more slowly, more massive NS cool faster.

As we have mentioned, for the sake of simplicity we did not include hyperonization and other possibilities, like kaon condensation and fermion condensation, which may stimulate a more rapid cooling, as they are working in the same direction as pion condensation. We did not include internal heating which would yield a slower cooling. We did not include possible quark matter effects. The latter need a special treatment. The possibility of color superconductivity in dense NS interiors opens a number of interesting possibilities like the so called two-flavor color superconductivity (2SC) phase, color-flavorlocking (CFL) phase, and color-spin-locking (CSL) phase. Their cooling is essentially different. We will return to this discussion in the near future.

Acknowledgements. H.G. and D.V. acknowledge the hospitality and support of Rostock University. The work of H.G. has been supported in part by the Virtual Institute of the Helmholtz Association under grant No. VH-VI-041, that of D.V. has been supported in part by DFG (project 436 RUS 17/117/03). We thank E.E. Kolomeitsev for the help and discussions and S. Popov for discussions concerning the results.

\section{References}

Akmal, A., Pandharipande, V. R., \& Ravenhall, D. G. 1998, Phys. Rev. C, 58,1804

Bahcall, J. N., \& Wolf, R. A. 1965, Phys. Rev. B, 140, 1445

Baiko, D. A., Haensel, P., \& Yakovlev, D. G. 2001, A\&A, 374, 151

Blaschke, D., Röpke, G., Sedrakian, A. D., Schulz, H., \& Voskresensky, D. N. 1995, MNRAS, 273, 596

Blaschke, D., Klähn, T., \& Voskresensky, D. N. 2000, ApJ, 533, 406

Blaschke, D., Grigorian, H., \& Voskresensky, D. N. 2001, A\&A, 368 561

Brown, G. E., Kubodera, K., Page, D., \& Pizzochero, P. 1988, Phys. Rev. D, 37, 2042

Ericson, T. O., \& Weise, W. 1988, Pions in Nuclei (Oxford: Clarendon)

Flowers, E., Ruderman, M., \& Sutherland, P. 1976, ApJ, 205, 541

Friman, B., \& Maxwell, O. V. 1979, ApJ, 232, 541

Glen, G., \& Sutherland, P. 1980, ApJ, 239671

Glendenning, N. K. 1992, Phys. Rev. D, 461274

Gnedin, O. Y., \& Yakovlev, D. G. 1995, Nucl. Phys. A, 582, 697

Heiselberg, H., \& Hjorth-Jensen, M. 1999 [arXiv: astro-ph/9904214]

Ivanov, Yu. B., Knoll, J., van Hees, H., \& Voskresensky, D. N. 2001, Phys. Atom. Nucl., 64, 652

Iwamoto, N. 1982, Ann. Phys., 141, 1

Kaminker, A. D., Yakovlev, D. G., \& Gnedin, O. Y. 2002, A\&A, 383, 1076

Kolomeitsev, E. E., \& Voskresensky, D. N. 2003, Phys. Rev. C, 68, 015803

Lattimer, J. M., Pethick, C. J., Prakash, M., \& Haensel, P. 1991, Phys. Rev. Lett., 66, 2701

Leinson, L. B. 2000, Phys. Lett. B, 473, 318

Lombardo, U., \& Schulze, H.-J. 2001, in Physics of Neutron Stars Interiors, ed. D. Blaschke, N. K. Glendenning, \& A. Sedrakian, Lect. Notes Phys., 578, 30

Lyne, A. G., Burgay, M., Kramer, M., et al. 2004 [arXiv: astro-ph/0401086]

Maruyama, T., Tatsumi, T., Voskresensky, D. N., Tanigawa, T., \& Chiba, S. 2003 [arXiv:nucl-th/0311076]
Maruyama, T., Tatsumi, T., Voskresensky, D. N., et al. 2004 [arXiv:nucl-th/0402002]

Maxwell, O. V. 1979, ApJ, 231, 201

Maxwell, O., Brown, G. E., Campbell, D. K., Dashen, R. F., \& Manassah, J. T. 1977, ApJ, 216, 77

Migdal, A. B., Saperstein, E. E., Troitsky, M. A., \& Voskresensky, D. N. 1990, Phys. Rep., 192, 179

Migdal, A. B. 1959, ZhETF, 37, 249

Nomoto, K., \& Tsuruta, S. 1981, ApJ Lett., 250, 19

Popov, S. B. 2004 [arXiv: astro-ph/0403710]

Rajagopal, K., \& Wilczek, F. 2000, in At the frontier of particle physics, ed. M. Shifman (World Scientific), 3, 2061

Ravenhall, D. G., Pethick, C. J., \& Wilson, J. R. 1983, Phys. Rev. Lett., 50, 2066

Schaab, Ch., Voskresensky, D., Sedrakian, A. D., Weber, F., \& Weigel, M. K. 1997, A\&A, 321, 591

Schulze, H.-J., Cugnon, J., Lejeune, A., Baldo, M., \& Lombardo, U. 1996, Phys. Lett. B, 375, 1

Schwenk, A., \& Friman, B. 2004, Phys. Rev. Lett., 92, 082501

Shapiro, S., \& Teukolsky, S. A. 1983, Black Holes, White Dwarfs and Neutron Stars: The Physics of Compact Objects (New York: Wiley ), Chap. 11

Suzuki, T., Sakai, H., \& Tatsumi, T. 1999 [arXiv: nucl-th/9901097]

Takatsuka, T., \& Tamagaki, R. 1997, Prog. Theor. Phys., 97, 345

Takatsuka, T., \& Tamagaki, R. 2004, Prog. Theor. Phys., in press [arXiv: nucl-th/0402011]

Tatsumi, T. 1988, Prog. Theor. Phys., 88, 22

Taylor, J. H., \& Weisberg, J. M. 1989, ApJ, 345, 434

Thorsett, S. E., \& Chakrabarty, D. 1999, ApJ, 512, 288

Tsuruta, S. 1979, Phys. Rep., 56, 237

Tsuruta, S. 2004, in Young Neutron Stars and their Environments, ed. F. Camilo, \& B. M. Gaensler, Proc. IAU Symp., 218 [arXiv: astro-ph/0401245]

Tsuruta, S., \& Cameron, A. G. W. 1965, Canad. J. Phys., 43, 2056

Tsuruta, S., Teter, M. A., Takatsuka, T., Tatsumi, T., \& Tamagaki, R. 2002, ApJ, 571, L143

Umeda, H., Nomoto, K., Tsuruta, S., Muto, T., \& Tatsumi, T. 1994, ApJ, 431, 309

Voskresensky, D. N. 1997, Phys. Lett. B, 392, 262

Voskresensky, D. 2001, in Physics of Neutron Stars Interiors, ed. D. Blaschke, N. K. Glendenning, \& A. Sedrakian, Lect. Notes Phys., 578,467

Voskresensky, D. N., \& Mishustin, I. N. 1982, Sov. J. Nucl. Phys., 35, 667

Voskresensky, D. N., \& Senatorov, A. V. 1984, JETP Lett., 40, 1212

Voskresensky, D. N., \& Senatorov, A. V. 1986, JETP, 63, 885

Voskresensky, D. N., \& Senatorov, A. V. 1987, Sov. J. Nucl. Phys., 45, 411 (Senatorov, A. V., \& Voskresensky, D. N. 1987, Phys. Lett. B, 184, 119)

Voskresensky, D. N., Kolomeitsev, E. E., \& Kämpfer, B. 1998, JETP, 87, 211

Voskresensky, D. N., Khodel, V. A., Zverev, M. V., \& Clark, J. W. 2000, ApJ, 533, L127

Voskresensky, D. N., Yasuhira, M., \& Tatsumi, T. 2002, Phys. Lett. B, 541, 93; Nucl. Phys. A, 723, 291

Wambach, J. 2003, Prog. Part. Nucl. Phys., 50, 615

Wiringa, R. B., Stoks, V. G., \& Schiavilla, R. 1995, Phys. Rev. C, 51, 38

Yakovlev, D. G., Gnedin, O. Y., Kaminker, A. D., Levenfish, K. P., \& Potekhin, A. Y. 2004a, Adv. Space Res., 33, 523

Yakovlev, D. G., Levenfish, K. P., Potekhin, A. Y., Gnedin, O. Y., \& Chabrier, G. 2004b, A\&A, 417, 169 